\title{
Forward-Looking Behavior in Hawk-Dove Games in Endogenous Networks: Experimental Evidence ${ }^{\text {th }}$
}

\author{
Siegfried K. Berninghaus ${ }^{\mathrm{a}}$, Karl-Martin Ehrhart ${ }^{\mathrm{a}}$, Marion Ott ${ }^{\mathrm{a}, *}$ \\ ${ }^{a}$ Karlsruhe Institute of Technology (KIT), ETS, Zirkel 2, Building 20.21, 76131 Karlsruhe, Germany
}

\begin{abstract}
We conduct an experiment in continuous time: every subject can change her links to others and her action in a Hawk-Dove game, which she plays bilaterally with each of her linked partners, at any time. We hypothesize that norms exist regarding who establishes and thus pays for links, and that players take these norms into account when deciding on their strategy. For such limitedly forward-looking players who consider others' linking reactions we introduce a strategy adaptation rule (anticipatory better-reply rule, ABR) and a related stability criterion (reaction-anticipatingly stable, RAS). Our data support our assumption on linking reactions. Subjects seem to take these reactions into account when deciding to switch from Hawk to Dove (ABR-behavior). However, better-reply behavior is prevalent when short-term profits allure. RAS configurations occur more often than Nash equilibria of the base game; however, with respect to stability they perform similar.
\end{abstract}

Keywords: Hawk-Dove game, chicken game, local interaction, network formation, network experiment, forward-looking players

JEL classification: C72, C91, C92, D85

\section{Introduction}

What guides people in forming a social network if pairwise interaction is basically competitive? To find out, we design an experiment in which we embed a symmetric $2 \times 2$ normal-form game in an endogenously determined interaction structure. In this combined game players can establish links to others and choose an action for the $2 \times 2$

\footnotetext{
NOTICE: this is the authors' version of a work that was accepted for publication in Games and Economic Behavior. Changes resulting from the publishing process, such as peer review, editing, corrections, structural formatting, and other quality control mechanisms may not be reflected in this document. Changes may have been made to this work since it was submitted for publication. A definitive version was subsequently published in Games and Economics Behavior, DOI 10.1016/j.geb.2011.12.003.

*Corresponding author. Present address: RWTH Aachen University, Templergraben 64, 52062 Aachen, Germany. Telephone: +49 241 80-96 157. Fax: +49 241 80-92 345.

Email addresses: siegfried.berninghaus@kit.edu (Siegfried K. Berninghaus), ehrhart@kit.edu (Karl-Martin Ehrhart), marion.ott@rwth-aachen.de (Marion Ott)
} 
game which they play with each of their linked partners. For the player who establishes the link the interaction involves costs. Competition is represented as pairwise play of a game of opposing interests, namely the Hawk-Dove (HD) game, whereby a player chooses the same action $H$ (Hawk) or $D$ (Dove) for all his pairwise HD games.

The rules for link building relate to the approach of Bala and Goyal (2000). That is, a link causes costs for the player who establishes the link and two players are linked if at least one of them establishes a link to the other. Since all payoffs in our HD game are positive and only the player who establishes a link pays for it, we assume that a player always agrees to interact if another player establishes a link to her. ${ }^{1}$ We vary the link cost between our treatments.

The experiment is conducted in continuous time. Subjects can change their HDgame action and their links at any time and are able to instantaneously observe their opponents' HD-game actions and links. Simultaneous changes hardly ever occur and, thus, a subject knows all links and actions in the group directly before and after her changes. In contrast, when subjects make decisions in discrete rounds, simultaneous HD-game action and/or link changes by several subjects are likely to lead to frequent coordination failures; the continuous time design allows the avoidance of these failures.

The strategic interaction between two linked players is modeled by the HD game. ${ }^{2}$ In its two asymmetric equilibria in pure strategies one player acts amicably (Dove behavior) and the other acts exploitatively (Hawk behavior). ${ }^{3}$ This game represents a "contest in which each party is trying to prevail over the other" (Snyder, 1971) because each player prefers the equilibrium in which she plays $H$.

The two equilibria and the combination of two $D$ s are associated with efficient outcomes (i.e. maximum sum of payoffs). Thus, an efficient outcome in the HD game with endogenous network formation (HDN game) with more than two players, in which a player chooses one action for all pairwise HD games, allows for a maximum of only one $H$ since pairs of $H$ s are sources of inefficiency.

As an example for the HDN game consider bilateral negotiations within organizations in which people exhibit either amicable or exploitative negotiation behavior (e.g., Tinsley et al., 2002). ${ }^{4}$

Although a $D$ earns a lower payoff than an $H$ when they interact in a pairwise HD game, a $D$ is more attractive for others as an interaction partner than an $H$ in the HDN game. More $D$ s in the network increases potential payoffs for $H \mathrm{~s}$, but with too many

\footnotetext{
${ }^{1}$ An alternative assumption is that both players have to agree to have a link and both bear the cost (Jackson and Wolinsky, 1996).

${ }^{2}$ The HD game is a form of the chicken game and is rooted in evolutionary game theory (see Maynard Smith, 1982, for details).

${ }^{3}$ The HD game also has a symmetric equilibrium in mixed strategies.

${ }^{4}$ The HDN game can also be applied to confrontations in the context of a political crisis as bilateral coercion and bilateral bargaining under the threat of violence (e.g., Lipman, 1986; Snyder, 1971; Cornell and Roll, 1981).
} 
$H$ s in the network, $D$ becomes the better action. In the experiment, subjects have to deal with these different opposing effects.

Our aim was to learn about the interplay of network formation and pairwise strategic interaction. Do subjects form a network and adjust their actions in the HD game (H or $D$ ) taking this network as given, or do they first choose an action and choose the appropriate partners for interaction afterwards? Hence, do we observe stable networks and action dynamics or stable action configurations and evolving network, or do both strategic elements vary a lot? Are there regularities in behavior? Do subjects adjust to the given situation, i.e. can their behavior be captured by the better-reply rule, and do we observe stabilization in certain states, e.g. in a Nash equilibrium of the HDN game? Or should we think of other criteria that might be better suited for our game, in the way that players take the impact of their choices on others into consideration?

Considering the characteristics of our repeated HDN game, we develop the following hypotheses. Since a player's link only affects her bilateral interaction with one other player, while her HD-game action affects all interactions with other players, we expect that the network will be adjusted to the choices of action, rather than the other way round. Hence, we expect subjects to adjust links rather than HD-game actions in response to another subject's change of action. Consequently, a subject who changes her HD-game action should foresee others' link reactions. So, in addition to testing the better-reply behavior rule (BR) and the Nash equilibrium (NE), we introduce another behavioral rule (anticipatory better-reply, ABR) and the related stability criterion (reaction-anticipating stability, RAS) that take this foresight into account.

In the experiment there is a lot of dynamics. Here, we observe many more link changes than HD-game action changes and the subjects indeed tend to adjust their links to the action configuration. Moreover, there is evidence that subjects take these link adjustments into account when making their decisions (as postulated by the ABR) and predominantly stay in or nearby configurations that meet the RAS criterion. This implies that subjects also consider their attractiveness to others when deciding on whether to opt for an $H$ or a $D$ action. They switch to amicable $D$ behavior when this attracts many partners. However, we also observe attempts to exploit partners by switching to $H$; a behavior in line with the BR, which turns out to bring only short-term profits.

Finally, we find that subjects' payoffs from playing amicably (as a $D$ ) are on average higher than those from playing exploitatively (as an $H$ ). More $D$ s enable high total payoffs, which favors $D$ s on average.

To the best of our knowledge, this is the first experimental investigation of endogenous interaction in the HD game. Our research is most closely related to the work of Corbae and Duffy (2008) and Corten and Buskens (2010), who investigate coordination games and network formation, and that of Riedl and Ule (2002), who study network formation and prisoner's dilemma games. ${ }^{5}$ There are numerous differences, however: in

\footnotetext{
${ }^{5}$ Kosfeld (2004) gives an overview of experiments on coordination games with different fixed inter-
} 
addition to different $2 \times 2$ games, link-cost structures, link-establishing processes, and other components, these authors conducted their experiments in discrete time, i.e. in rounds. ${ }^{6}$ The HD game is examined experimentally by Neugebauer et al. (2008), who find that most subjects act exploitatively rather than amicably.

Bramoullé et al. (2004) and Berninghaus and Vogt (2006) provide a theoretical analysis of $2 \times 2$ games in endogenous networks for our environment. Related models with coordination games can be found in Jackson and Watts (2002), Skyrms and Pemantle (2000), and Hojman and Szeidl (2006); Ule (2005) analyzes the prisoner's dilemma.

In the next section we describe the theoretical model. Before we introduce and discuss behavioral hypotheses and stability criteria in Section 4, we present the design of our experiment in Section 3. In Section 5, we analyze the experimental results with respect to our theoretical hypotheses and Section 6 concludes.

\section{The HD Networking Game (HDN Game)}

Consider a set of players $I=\{1, \ldots, n\}, n \geq 3$, who create their interaction structure via unilaterally costly links and who each choose one action in a $2 \times 2$ normal-form game played with each of their interaction partners. In the following, we describe the $2 \times 2$ game separately from the game of choosing interaction partners. Then we combine both approaches and formulate the HD networking game (HDN game). ${ }^{7}$

Table 1: Generalized Hawk-Dove Game

\begin{tabular}{ccc} 
& $H$ & $D$ \\
\hline$H$ & $d, d$ & $a, c$ \\
\hline$D$ & $c, a$ & $b, b$ \\
\hline$a>b>c$ & $>d$
\end{tabular}

If two players $i$ and $j$ are linked, they play the symmetric $2 \times 2$ normal-form game $\Gamma=\{\{i, j\}, \Sigma, \pi(\cdot)\}$ with strategy set $\Sigma:=\{H, D\}$, where $H$ represents the Hawk strategy and $D$ the Dove strategy. The values of the $H D$ payoff function $\pi(\cdot): \Sigma \times \Sigma \rightarrow \mathbb{R}$ are shown in Table 1. This game is a generalized HD game (or chicken game) derived from the traditional HD game of Maynard Smith (1982). In what follows, a strategy in the HD game (i.e. $H$ or $D$ ) is called action. The HD game has three Pareto-optimal

action structures and experiments on network formation and the related theory.

${ }^{6}$ In Riedl and Ule's experiment (2002), subjects simultaneously choose links and prisoner's dilemma actions in each of 60 rounds. Corbae and Duffy (2008) set up a two-stage game consisting of a networkdetermining stage and a five-period segment in which coordination game actions are chosen. In Corten and Buskens (2010), 15 rounds are played, each consisting of three stages in which subjects are allowed to: change their links, accept/reject links unilaterally proposed by others, and choose their actions in the coordination game.

${ }^{7}$ A related class of games consists of so-called "social games" (Jackson and Watts, 2008, 2010). 
payoff combinations $(a, c),(c, a)$, and $(b, b)$, of which the first two refer to the asymmetric Nash equilibria $(H, D)$ and $(D, H)$. A player who chooses $D$ is better off when matched with another $D$ rather than with an $H$, although $(D, D)$ is no equilibrium of $\Gamma$.

The interaction structure is not fixed but is determined by individual decisions. Player $i$ 's strategy in the network game in normal-form is a vector of ones and zeros, $g_{i} \in\{0,1\}^{n}$. If $g_{i j}=1$, then player $i$ establishes a link to player $j$, otherwise $g_{i j}=0$. If at least one of two players has a link to the other one they are linked (we also say they have a link). Linked players play the HD game $\Gamma$. A player cannot establish a link to herself, i.e. $g_{i i}=0$ for all $i$, and cannot play the game with herself. A single link is said to exist if only one of two players has a (unilateral) link and a double link means that they both establish links, i.e. they are bilaterally linked. In the latter instance, one link is redundant because a pair with a double link plays only one HD game. Each strategy configuration $g=\left(g_{1}, \ldots, g_{n}\right)$ in the network game generates a directed graph, denoted by $\mathcal{G}_{g}$, whose vertices represent players and a directed edge between $i$ and $j$ (i.e. $g_{i j}=1$ ) indicates that $i$ has a link to $j$. The set of $i$ 's neighbors in a network $\mathcal{G}_{g}$ is denoted by $N_{i}\left(\mathcal{G}_{g}\right)$ and defined as the union of the set of all players to whom $i$ has a link (i.e. $g_{i j}=1$ ) and the set of all players who establish a link to $i$ (i.e. $g_{j i}=1$ ): $N_{i}\left(\mathcal{G}_{g}\right):=\left\{j \mid \max \left\{g_{i j}, g_{j i}\right\}=1\right\}$. The set of $i$ 's neighbors to whom $i$ has a link, $N_{i}^{a}\left(g_{i}\right):=\left\{j \mid g_{i j}=1\right\}$, depends solely on $i$ 's network strategy vector $g_{i}$. Establishing a link to another player is costly. For simplicity, we assume that cost $k>0$ per link is constant and identical for all players.

Combining the two components, the strategic situation of a player is modeled as a non-cooperative game in normal-form, which is called HD networking game (HDN game). In this game an individual decision is composed of one action $\sigma_{i} \in\{H, D\}$ for the HD games and the choice of neighbors via links $g_{i} \in\{0,1\}^{n}$ in the network game. The number of players choosing $H$ and $D$ are denoted by $n_{H}$ and $n_{D}$ with $n_{H}+n_{D}=n$.

The HDN game is denoted by $G=\left(I ; S_{1}, \ldots, S_{n} ; P_{1}(\cdot), \ldots, P_{n}(\cdot)\right)$ with strategy sets $S_{i}:=\{H, D\} \times\{0,1\}^{n}$ and payoff functions $P_{i}: S_{1} \times \ldots \times S_{n} \rightarrow \mathbb{R}$. Each strategy configuration $s=\left(s_{1}, \ldots, s_{n}\right)=\left(\left(\sigma_{1}, g_{1}\right), \ldots,\left(\sigma_{n}, g_{n}\right)\right)$ induces a vector of each player's action in her HD games and a network $\mathcal{G}_{g}$. Player $i$ 's payoff function is

$$
P_{i}(s):=\sum_{j \in N_{i}\left(\mathcal{G}_{g}\right)} \pi\left(\sigma_{i}, \sigma_{j}\right)-k\left|N_{i}^{a}\left(g_{i}\right)\right|,
$$

where $\left|N_{i}^{a}\left(g_{i}\right)\right|$ is the cardinality of $N_{i}^{a}\left(g_{i}\right)$. A player's payoff depends on her HD-game action and the actions of the players with whom she is linked minus her total link cost. According to our payoff definition, player $i$ may benefit from being linked with $j$ even though she does not establish the link (if $g_{i j}=0$ but $g_{j i}=1$ ). A player $i$ 's payoff from a link either equals her HD payoff $\pi\left(\sigma_{i}, \sigma_{j}\right)$ (if $g_{i j}=0$ but $g_{j i}=1$ ) or $\pi\left(\sigma_{i}, \sigma_{j}\right)-k$ (if $\left.g_{i j}=1\right)$. In what follows, the term strategy refers to a strategy in the HDN game $G$. 


\section{Experimental Design}

In our experiment, the parameters of the HD game in Table 1 are chosen as $a=80$, $b=60, c=40$, and $d=20$ (Table 2). This game is embedded in a HDN game with $n=6$ agents, as described in Section 2. We consider three treatments that differ with respect to link cost $k=30, k=50$, and $k=70$. The corresponding treatments are denoted by HD30, HD50, and HD70 (Table 3). The particular values of $k$ influence the profitability of links. In each treatment, we run six independent groups, each with six subjects. The experimental instructions can be found in Appendix C.

Table 2: Payoff Table of the Hawk-Dove Game in the Experiment

\begin{tabular}{ccc} 
& $H$ & $D$ \\
\hline$H$ & 20,20 & 80,40 \\
\hline$D$ & 40,80 & 60,60 \\
\hline
\end{tabular}

The HDN game is repeated in continuous time. Each game lasts exactly 30 minutes and starts after all subjects have made their first decision, i.e. after each subject has decided on links and the action. Thereafter, subjects can change single components of their strategies at any time, i.e. whenever they want, they select a link or the action and confirm its change. Information is updated 10 times per second. The current payoff flows, i.e. the current values of the subjects' payoff functions $P_{i}(s)$, are computed every tenth of a second and a subject's accumulated payoffs are given as her "integrated" payoff flows up to the given moment. The information on every subject's screen includes the elapsed time, her current payoff flow, and her accumulated payoff. A subject's own and the other subjects' links are illustrated graphically on the screen by directed arrows, and the chosen actions $H$ or $D$ are indicated at the vertices of the graph. ${ }^{8}$ The subjects with whom one is linked are shown in a different color from the remaining subjects.

Payoff flow and link cost are measured in experimental currency units (ExCU) per minute. For example, a subject who extracts a payoff flow of $140 \mathrm{ExCU}$ per minute for 15 seconds and then changes her strategy choice to obtain 200 ExCU per minute for 9 seconds receives for these 24 seconds an accumulated payoff of $(140 / 60) \cdot 15+(200 / 60)$. $9=65$ ExCU. Payoffs are accumulated over 30 minutes and paid out in cash after the experiment. Since negative payoffs are possible, we pay a show-up fee of 500 ExCU. Because of different potential earnings in the treatments, we vary the conversion rate. ${ }^{9}$

The experiment was conducted at the University of Karlsruhe as a computerized laboratory experiment. It was organized into nine sessions each with two groups. Before

\footnotetext{
${ }^{8}$ In the actual experiment the two actions were denoted by the neutral letters $X$ and $Y$.

${ }^{9}$ The conversion rate is $€ 1$ per $400 \mathrm{ExCU}$ in HD30, $€ 1$ per $300 \mathrm{ExCU}$ in HD50, and $€ 1$ per $170 \mathrm{ExCU}$ in HD70. The conversion rates are determined according to the payoff sums that can be reached in NE with three $H \mathrm{~s}$ and three $D$ s in the different treatments (see Table 4).
} 
Table 3: Experimental Treatments

\begin{tabular}{cccc}
\hline Treatment & Link cost $k$ & Number of group members $n$ & Number of groups \\
\hline HD30 & 30 & 6 & 6 \\
HD50 & 50 & 6 & 6 \\
HD70 & 70 & 6 & 6 \\
\hline
\end{tabular}

the experiment started, subjects had to solve a few problems to ensure that they understood the rules of the game. Participants were randomly selected from a data pool, in the laboratory subjects sat separated from each other, their seats were determined randomly, they were made anonymous via randomly assigned numbers, payment occurred individually, the twelve participants per session were distributed over two rooms in a way such that they could not conclude who is in their group. The average, minimum, and maximum payoffs earned were $€ 14.16$, $€ 8.85$, and $€ 22.27$, respectively. The average payoffs in HD30, HD50, and HD70 were $€ 14.63$, $€ 14.95$, and $€ 12.85$, respectively.

\section{Theoretical Considerations and Hypotheses}

First, as a natural benchmark for our repeated HDN game in continuous time, we consider the Nash equilibria of the HDN base game $G$.

Definition 1 (Nash equilibrium NE). A strategy configuration $s^{*}=\left(\left(\sigma_{1}^{*}, g_{1}^{*}\right), \ldots,\left(\sigma_{n}^{*}, g_{n}^{*}\right)\right)$ is a Nash equilibrium of $G$ if $P_{i}\left(s_{i}^{*}, s_{-i}^{*}\right) \geq P_{i}\left(s_{i}, s_{-i}^{*}\right)$ for all $s_{i} \in S_{i}$ and for all $i \in I$.

In a NE, no player has an incentive to change her links $g_{i}^{*}$, her action $\sigma_{i}^{*} \in\{H, D\}$, or both unilaterally. The NE for the HDN game $G$ are described in Bramoullé et al. (2004) and Berninghaus and Vogt (2006) (see Appendix A, Table A.11). The NE of our particular game $G$ (with the HD game of Table 2) are shown in Table A.13. The concept of a NE is closely tied to the better-reply rule (e.g. Friedman and Mezzetti, 2001).

Definition 2 (Better-Reply Rule BR). A player who deviates from her current strategy $s_{i}=\left(\sigma_{i}, g_{i}\right)$ changes to a strategy that would strictly increase her payoff $P_{i}(\cdot)$ (in the $H D N$ game $G$ ) if the other players do not change their strategies.

The $\mathrm{BR}$ relates to $\mathrm{NE}$ in the sense that in a NE no player can improve her payoff according to the BR. The BR (as an individual behavior rule) and NE (as a stability criterion) are the first benchmarks for our experimental analysis. Before presenting the analysis, we will discuss and criticize these concepts as benchmarks for our repeated HDN game in continuous time and propose competing hypotheses. ${ }^{10}$

\footnotetext{
${ }^{10}$ Alternatively, we may use the subgame-perfect equilibrium as solution concept. Under the assumption of immediate reactions to link and action changes in players' strategy plans for the repeated game, the (subgame-perfect) folk theorem for infinitely repeated games in discrete time also applies to
} 

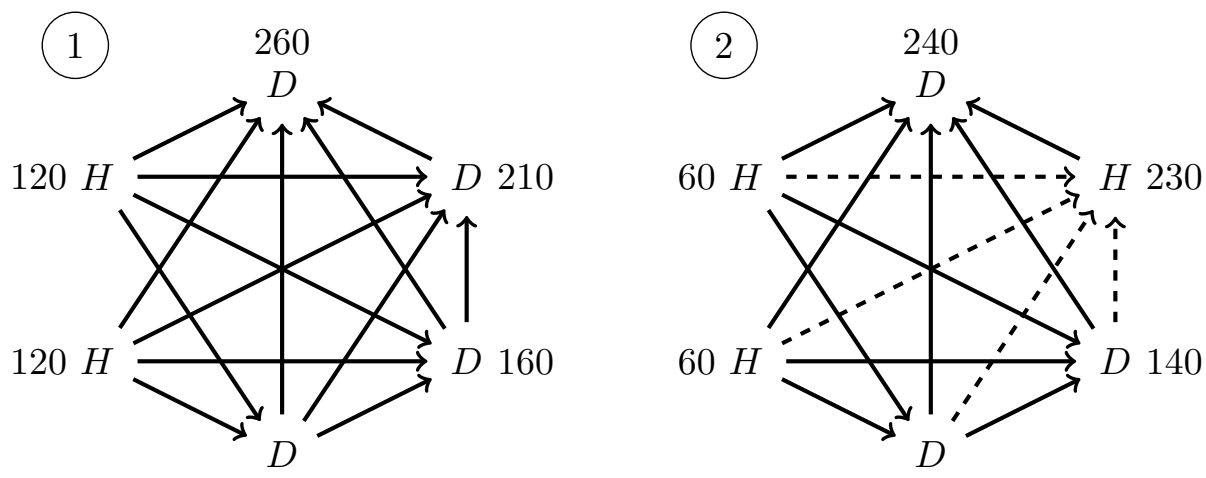

110
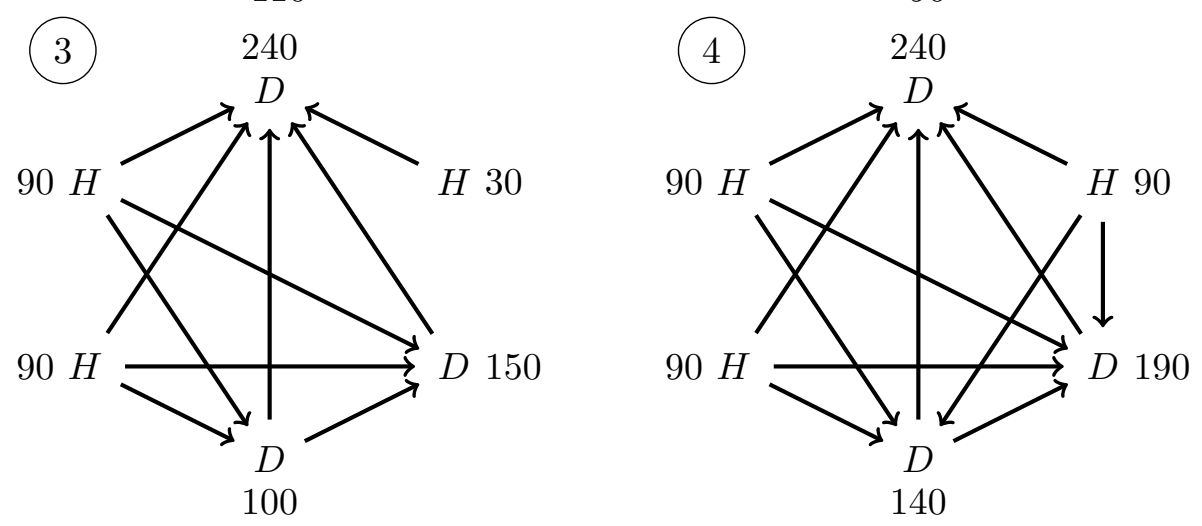

Figure 1: An example demonstrating the implausibility of NE as a solution concept $(k=50)$

NE of the base game $G$ and the BR imply the belief that other players will not react to one's own strategy change, i.e. they will keep their links and actions. But there exist many strategy configurations in which the profitability of an action change from $D$ to $H$ crucially depends on links established by others whose payoff from these links becomes negative after the action change. Hence, a change from $D$ to $H$ in accord with the BR may become unprofitable if the others react to this action change by deleting those links.

We exemplify this argument in Figure 1 for $k=50$. The first graph shows a network in which all single links with a positive payoff are established. An arrow pointing from an $H$ to a $D$ indicates that $H$ establishes the link and vice versa. The players' payoffs are given next to their action choice. The second graph shows the same configuration except for the northeastern player, who changes her action from $D$ to $H$ and thereby

repeated continuous-time finite-horizon games (Ehrhart, 1997; Simon and Stinchcombe, 1989): every payoff combination within the convex hull of all feasible payoff combinations of the stage game that assigns to each player at least her minimax payoff is reachable as an average payoff via a subgame-perfect equilibrium path of the repeated game in continuous time. Thus, we face a huge range of equilibrium payoffs, most of them constituted by complex combinations of strategy plans. For these reasons, we discard the subgame-perfect equilibrium concept as a benchmark for our experimental data. 
increases her payoff from 210 to 230 . Hence, the first graph is not a NE. This action change, however, causes four other players' payoff from their link to the northeastern player to become negative (indicated by dashed arrows in Graph 2). Therefore, the northeastern player may expect that those links will be deleted, as shown in Graph 3 . Her best response to these reactions is to establish a link to the southern $D$ and to the southeastern $D$ (Graph 4), resulting in a payoff of 90, which is below her primary payoff of 210. Hence, if the player expects these reactions, she compares 210 with 90 and thus has no incentive to deviate to $H$. Therefore, non-NE configurations might appear stable, which makes Nash stability questionable in the context of the HDN game.

Similarly, the BR and NE do not capture a player's expectation that opponents will establish profitable links, even if establishing the link is not profitable for the player herself. For example, at $k=50$, establishing a link between $H$ and $D$ is only profitable for $H$. Consider a player who unilaterally deviates from a NE by changing her action from $H$ to $D$. If this player anticipates that the other $H$ s will afterwards establish links to her, her action change may become profitable. It turns out that almost every NE in our game is vulnerable to deviations by players who expect adequate link adaptations by others after an action change. Thus, in this sense NE might be unstable.

This criticism motivates us to consider a special type of forward-looking behavior where decisions are based on beliefs that take account of the described credible individual threats and promises of link adaptation. We formalize these beliefs in Definition 4. Then, we introduce a behavioral rule that imposes better responses given our belief rule (Definition 5). Next, we define the stability criterion (Definition 6) according to that a strategy configuration is stable if no player with our beliefs has a better response. The beliefs about link adjustments, the behavioral rule, and the stability criterion are called Expected Linking Reactions (ER), Anticipatory Better-Reply Rule (ABR), and Reaction-Anticipatingly Stable (RAS), respectively. The comparison of these criteria with BR and NE consists of three pairs: ER versus the belief that the other players keep their strategies, the ABR (i.e. better responses to our beliefs) versus the BR, and our stability criterion RAS versus the NE.

Remember that a player's links and her action in the HDN game have different implications: a link affects only the bilateral interaction with one other player, whereas the action affects all of her interactions. Accordingly, an action adjustment in response to another player's strategy change is likely to provoke the reactions of others, while the direct consequences of a link adjustment are easier to foresee since it only affects one other player. Thus, we presume that a player is more likely to react to another player's strategy change by deleting or establishing a link than by changing her action, and will expect others to behave in the same way.

To come up with a general rule of link adjustment (Definition 4) we first introduce a notation to distinguish links.

Definition 3 (Unambiguous, Ambiguous, and Unfavorable Links). A link established by player $i$ to player $j$ is called 
- unambiguous for player $i$ if she obtains a positive payoff from this link ${ }^{11}$ and

- i's payoff from this link is higher than or equal to $j$ 's HD payoff or

- if $j$ would have a negative payoff from this link if he established it;

- ambiguous for player $i$ if she obtains a positive payoff from this link smaller than j's HD payoff and if this also applies vice versa (i.e. if $j$ established the link, he would receive a positive payoff smaller than $i$ 's $H D$ payoff);

- unfavorable for player $i$ if she obtains a negative payoff from this link or if the link would be unambiguous for $j$ if he established it.

In this sense, unambiguous and ambiguous links are favorable links. With our parameters, a link from $H$ to $D$ is always unambiguous because the $H$ gets a positive payoff if she establishes the link (50 in HD30, 30 in HD50, 10 in HD70), while the $D$ obtains a lower payoff than the $H$ in HD30 $(40<50)$ and would suffer a loss if she established the link in HD50 and HD70 (payoffs are -10 and -30). ${ }^{12}$ Thus, a link from $D$ to $H$ is unfavorable for the $D$ in all treatments. According to the definition, an ambiguous link for $i$ would also be ambiguous for $j$ if she paid for it. Ambiguous links exist only between two $D$ s in HD30 and HD50. The $D$ who establishes the link receives a payoff of 30 or 10 , respectively, while the other $D$ obtains 60 in both treatments. In HD70 a link between two $D$ s is unfavorable, because the $D$ who establishes the link suffers a loss of 10 . Links between two $H$ s are always unfavorable (payoffs are -10 in HD30, -30 in HD50, -50 in HD70). For a general classification see Table B.15.

Based on the characterization of links in Definition 3, we employ the following hypothesis about expected linking reactions to a player's strategy change.

Definition 4 (Expected Linking Reactions ER). In response to player $i$ 's strategy change from $s_{i}=\left(\sigma_{i}, g_{i}\right)$ to $s_{i}^{\prime}=\left(\sigma_{i}^{\prime}, g_{i}^{\prime}\right)$ player $j(j \neq i)$ will establish an unambiguous link to $i$ if $g_{i j}^{\prime}=0$ (i.e. if $i$ has no link to $j$ ) and will delete an unfavorable link to $i$ and a double ambiguous link to $i$. Player $j$ will not do any other changes.

According to ER, players are expected to react to another player's strategy change solely by adapting their links (i.e. by establishing unambiguous links and deleting unfavorable and double ambiguous links). Players are not expected to change their action, establish double, unfavorable, and ambiguous links, and delete unambiguous and single ambiguous links. ER prescribe a unique response to any strategy change of another player.

\footnotetext{
${ }^{11}$ If player $i$ has established a link to player $j$ (i.e. $g_{i j}=1$ ), her payoff from this link is her HD payoff $\pi\left(\sigma_{i}, \sigma_{j}\right)$ (i.e. $i$ 's payoff from the HD game with $j$ ) minus the cost of this link $k$ (see Section 2).

${ }^{12}$ We consider the classification of links of $H$ to $D$ as "unambiguous" in HD50 and HD70 to be unproblematic. In HD30, alternative classification rules, such that an $H$ does not always have to pay for such links, come into consideration.
} 
With our parameters, all but one response in the definition of ER increase the reacting player $j$ 's payoff. ${ }^{13}$ That is, they reflect behavior according to the BR. Since ER allow for link changes only, assume inertia, and take the unambiguousness of links into account, there exist responses that are consistent with the BR but not expected by ER. Inertia means that players are expected to keep single ambiguous links but not to establish missing ambiguous links. Also, ER assume that players establish unambiguous links and keep (even double) unambiguous links. Hence, there are three types of link changes that are in line with the BR but not expected by ER. ${ }^{14}$ Therefore, except for the single case mentioned above, one can say that ER assume conservative BR responses.

We now define a behavioral rule for strategy changes, according to that a player takes ER into consideration.

Definition 5 (Anticipatory Better-Reply Rule ABR). A player who deviates from her current strategy $s_{i}=\left(\sigma_{i}, g_{i}\right)$ changes to a strategy that will strictly increase her payoff $P_{i}(\cdot)$ (in the HDN game $G$ ) if the other players react to this change according to ER.

We call this the anticipatory better-reply rule (ABR) since a player who intends to change her strategy expects her opponents to react according to ER. Thus, a player's change of her strategy is considered consistent with the ABR if the change increases her payoff, provided that the other players adapt their links according to ER. Due to the inertia assumption involved in ER, the ABR leaves it to the deviating player to establish missing ambiguous links herself. Note that the ABR is self-consistent: all link reactions that are in line with ER are also ABR-consistent strategy changes.

Both the BR and the ABR prescribe better-response behavior but they differ essentially with respect to the beliefs to that players respond (the others do nothing vs. the others adjust links according to ER). As a consequence, strategy changes that are in line with the BR are often not consistent with the ABR and the other way round. While most changes of links are in line with both rules, ${ }^{15}$ strategy changes that involve an action change drive the difference between the ABR and the BR. This is because a change of the action may trigger reactions by many players, and it alters the type (according to Definition 3) of existing or missing links. The profitability of a strategy change that involves an action change usually depends on others' link adjustments and, therefore, these changes are better suited to contrast the ABR and the BR than strategy changes

\footnotetext{
${ }^{13}$ The only exception is: in HD30, as a reaction to a strategy change of $i, j$ is expected to delete a single link to $i$ if $j$ is a $D$ and $i$ is an $H$.

${ }^{14}$ First, establishing an ambiguous link (i.e. a single link from $D$ to another $D$ in HD30 and HD50) is not expected by ER but is consistent with the BR. Second, establishing a single link from $D$ to $H$ in HD30 is in line with the BR but not with ER because it is an unfavorable link for $D$. Third, an $H$ deleting a double link to a $D$ is not expected by ER because it is an unambiguous link for $H$.

${ }^{15}$ There exists only one non-concordant case in all treatments and an additional case in HD30: In all treatments, deleting a double link between an $H$ and a $D$ by the $H$ is in line with the BR but not with the ABR, because the $H$ expects the $D$ to delete the link. In HD30, deleting a single link by a $D$ to an $H$ is in line with the ABR, because the $D$ expects the $H$ to establish the link, but not with the BR.
} 
that consist only of link changes. Also, such strategy changes are our main motivation for suggesting the ABR as an alternative behavioral rule. We therefore restrict our experimental analysis of the ABR and the BR to strategy changes that involve an action change.

Considering the close relationship between the BR and NE, we wonder whether there is a similar stability criterion characterizing network and action configurations that relates to the ABR. We will therefore formalize a stability criterion called reactionanticipating stability. The basic idea behind this criterion is similar to that behind the limited forecast equilibrium developed by Jehiel (2001). ${ }^{16}$

Definition 6 (Reaction-Anticipatingly Stable RAS). A strategy configuration $s^{* *}=\left(s_{1}^{* *}, \ldots, s_{n}^{* *}\right)=\left(\left(\sigma_{1}^{* *}, g_{1}^{* *}\right), \ldots,\left(\sigma_{n}^{* *}, g_{n}^{* *}\right)\right) \in S$ is reaction-anticipatingly stable if $P_{i}\left(s_{i}^{* *}, s_{-i}^{* *}\right) \geq P_{i}\left(s_{i}, E R_{-i}\left(s_{i}, s_{-i}^{* *}\right)\right)$ for all $s_{i} \in S_{i}$ and for all $i \in I$.

$E R_{-i}\left(s_{i}, s_{-i}^{* *}\right)$ denotes the strategy vector of all players $j \neq i$ after they adapt their links to player $i$ 's deviation to $s_{i}$ according to ER and subject to the strategy configuration $s_{-i}^{* *}$ that they play at the time player $i$ changes her strategy. ${ }^{17}$ Evidently, in a RAS configuration, no player has an incentive to deviate if the other players are expected to react according to ER. That is, the RAS criterion is based on the assumption that players act rationally with respect to ER and thus mirror forward-looking thinking. ${ }^{18}$ For a general characterization of RAS configurations, see Table A.12. Table A.14 gives an overview of all RAS configurations for the HDN games used in our experiment.

Table 4 contrasts NE and RAS configurations according to the ratio of number of $H \mathrm{~s}$ to that of $D \mathrm{~s}\left(n_{H}: n_{D}\right)$ and displays for each configuration the sum of players' payoffs. ${ }^{19}$ Dashes indicate configurations that cannot be reached via either NE or RAS. Payoffs in italics indicate configurations where the networks are not fully characterized by a description of links between types but need to satisfy additional restrictions on the number of links of $D$ s (see tables A.11 and A.12).

Except for the ratio of 2:4 in HD70, the two criteria do not coincide, i.e. RAS configurations always consist of more $D$ s than the NE. Graph 1 in Figure 1 is an example of a strategy configuration with four $D$ s that is RAS but not a NE. Moreover, RAS allows for a maximum of only two $H \mathrm{~s}$, whereas NE requires a minimum of three $H \mathrm{~s}$ in HD30 and HD50 and allows up to six Hs in HD50 and HD70. This result is in line with Jehiel (2001), who shows that a limited forecast, as the ABR postulates, induces players to

\footnotetext{
${ }^{16}$ However, there are crucial differences: In contrast to Jehiel (2001), payoffs that occur during the adaptation process are not taken into account (as our setting allows for fast adjustment, those payoffs are of minor relevance), and the length of the forecasting period (i.e. the number of adjustments) is endogenous in our model because it depends on the number of links that players expect to be adjusted.

${ }^{17}$ Since the ER only allows for link changes, players' actions in $E R_{-i}\left(s_{i}, s_{-i}^{* *}\right)$ are the same as in $s_{-i}^{* *}$.

${ }^{18}$ For some discussion of beliefs in repeated games, see e.g. Morris (1995), Section 5.1.

${ }^{19}$ The NE and RAS configurations are derived in Appendix A and Appendix B.
} 
Table 4: NE and RAS Configurations and Their Payoff Sums [ExCU per Minute]

\begin{tabular}{ccccccccc}
\hline & & \multicolumn{1}{c}{ Ratio of number of $H$ s to number of $D$ s $\left(n_{H}: n_{D}\right)$} \\
Treatment & Criterion & $0: 6$ & $1: 5$ & $2: 4$ & $3: 3$ & $4: 2$ & $5: 1$ & $6: 0$ \\
\hline HD30 & NE & - & - & - & 1080 & 810 & - & - \\
& RAS & - & $1350^{*}$ & 1260 & - & - & - & - \\
\hline HD50 & NE & - & - & - & 840 & 630 & 350 & 0 \\
& RAS & $1050^{*}$ & $1050^{*}$ & 980 & - & - & - & - \\
\hline HD70 & NE & - & - & 400 & 450 & 400 & 250 & 0 \\
& RAS & - & 250 & 400 & - & - & - & - \\
\hline
\end{tabular}

* efficient outcome (i.e. maximum possible payoff sum)

behave more cooperatively in repeated prisoner's dilemma games than NE predicts. ${ }^{20}$

Since the presence of more than one $H$ constitutes a source of inefficiency, the efficient outcome (i.e. maximum possible payoff sum) requires that all players but a maximum of one choose $D$ and all players are linked via single links (i.e. 15 links exist). ${ }^{21}$ Payoff sums in the efficient outcomes are 1350, 1050, and 750 ExCU per minute in HD30, HD50, and HD70, respectively. In HD30 and HD50, in which it is favorable for a $D$ to establish a link to another $D$, the efficient outcome can be achieved by RAS configurations with zero or one $H$ but not by NE. Since in HD70 a link from $D$ to $D$ is not profitable, the efficient outcome cannot be achieved by NE or RAS.

\section{Experimental Results}

In this section, we describe some observations (Section 5.1) and analyze the hypotheses ER, BR, and ABR as well as NE and RAS (Section 5.2). Throughout, we use a significance level of $5 \%$ and we apply two-tailed tests. In every statistical test, the average across all subjects/decisions in a group is used as an observation. Thus, for every test there are six independent observations per treatment. The group results, which correspond to the presented average results, can be found in the online supplementary material.

\subsection{Characteristics of Observed Decisions}

Table 5 presents some selected observations. In all treatments, we observe many more link changes than action changes (second and third columns). The average ratio is 5.8 to 1 . Since a player can change only one action but five links, one could argue that

\footnotetext{
${ }^{20}$ Ule (2005), however, finds that cooperative behavior in a finitely repeated prisoner's dilemma played in an endogenous interaction structure can be sustained in a subgame-perfect equilibrium.

${ }^{21}$ If $a+c=2 b$, which is fulfilled in our HD game, for every value of $n_{H}$ the maximum sum of payoffs is $\left(n_{H} n_{D}+\left(n_{D}\left(n_{D}-1\right) / 2\right)\right)(2 b-k)$ if $2 d \leq k \leq 2 b$ and $\left(n_{H} n_{D}+\left(n_{D}\left(n_{D}-1\right) / 2\right)\right)(2 b-k)+\left(n_{H}\left(n_{H}-\right.\right.$ $1) / 2))(2 d-k)$ if $0 \leq k \leq 2 d$, which is maximal for $n_{D}=n$ and $n_{D}=n-1$.
} 
Table 5: Characteristics of Link and Action Choices

\begin{tabular}{ccccccccccc}
\hline & \multicolumn{2}{c}{$\begin{array}{c}\text { Avg. number } \\
\text { of changes of }\end{array}$} & \multicolumn{2}{c}{$\begin{array}{c}\text { Avg. number } \\
\text { of }\end{array}$} & \multicolumn{2}{c}{$\begin{array}{c}\text { Avg. dwell time } \\
\text { [seconds] }\end{array}$} & \multicolumn{2}{c}{$\begin{array}{c}\text { Avg. payoff } \\
\text { [ExCU per min.] }\end{array}$} & $\begin{array}{c}\text { Avg. } \\
\text { efficiency }\end{array}$ \\
Treat. & links & actions & links & $H \mathrm{~s}$ & $D$ s & $H$ & $D$ & $H$ & $D$ & {$[\%]$} \\
\hline HD30 & 346 & 73 & 12.5 & 2.2 & 3.8 & 126 & 221 & 145.5 & 168.7 & 79.3 \\
HD50 & 425 & 71 & 12.0 & 2.0 & 4.0 & 116 & 204 & 108.3 & 133.2 & 75.9 \\
HD70 & 302 & 43 & 7.0 & 1.7 & 4.3 & 150 & 333 & 36.1 & 60.1 & 45.1 \\
\hline Avg. & 358 & 62 & 10.5 & 2.0 & 4.0 & 131 & 253 & 96.6 & 120.7 & 66.8 \\
\hline
\end{tabular}

the subjects change every component of their strategy vector with similar frequency. ${ }^{22}$ We do not find significant differences between the treatments in terms of the number of link changes or action changes. ${ }^{23}$ Due to the high number of action changes, around half of them are from $H$ to $D$ and half are from $D$ to $H .^{24}$

With regard to the average number of links in a group (fourth column of Table 5), we find significant differences between HD30 and HD70 and between HD50 and HD70. ${ }^{25}$ This pattern can be understood by considering the profitability of links. The difference between HD30 and HD50 is that in HD30 the link from $D$ to $H$ is profitable. This can yet be compensated for if the $H$ establishes the link to the $D$ in HD50. Because the numbers of $H \mathrm{~s}$ in both treatments are similar (see column five), the number of links should be similar too. We observe about two $H$ s and twelve links on average, which is a bit less than 14, the number of profitable links when there are two $H$. In HD70, however, only a link from $H$ to $D$ is profitable. Thus, fewer than eight links are profitable on average (four links of each of the approx. two $H \mathrm{~s}$ ), which is the number we observe.

We observe that $D$ is more often played than $H$ (fifth and sixth columns of Table 5) and that subjects' average dwell time in $D$ is longer than in $H$, i.e. subjects (on average) keep the $D$ action longer than the $H$ action before they change it (seventh and eighth columns). Both observations apply for all groups of all treatments. ${ }^{26}$ The comparison

\footnotetext{
${ }^{22}$ Comparing the number of changes per link, i.e. the number of link changes divided by five, and the number of action changes we cannot detect a difference. Sign test (pooled data of all 18 groups): 12 with more changes per link and 6 with more action changes, $p$-value $>0.05$.

${ }^{23}$ Comparison of average number of link changes: Overall comparison (Kruskal-Wallis test): test statistic $=2.351$, degrees of freedom $=2, p$-value $>0.05$.

Comparison of number of action changes: Overall comparison (Kruskal-Wallis test): test statistic $=$ 4.292 , degrees of freedom $=2, p$-value $>0.05$.

${ }^{24} \mathrm{~A}$ sequence of $x$ action changes by a player either consists of $x / 2$ changes in both directions (if $x$ is even) or of $(x+1) / 2$ changes in one direction and $(x-1) / 2$ changes in the other direction (if $x$ is odd). Thus, the numbers of a player's changes from $H$ to $D$ and from $D$ to $H$ are either equal or differ by one. Therefore, if $x$ is large, about half of the changes are from $H$ to $D$ and half are from $D$ to $H$.

${ }^{25}$ Comparison of average number of links: Overall comparison (Kruskal-Wallis test): test statistic $=$ 11.875, degrees of freedom $=2, p$-value $<0.05$. Pairwise multiple comparison (Tukey Test): HD30 vs. HD50: $p$-value $>0.05$; HD30 vs. HD70: $p$-value $<0.05$; HD50 vs. HD70: $p$-value $<0.05$.

${ }^{26}$ Sign tests (pooled data of all 18 groups): $p$-value $<0.05$.
} 
of the ratios of $H \mathrm{~s}$ to $D \mathrm{~s}$ with the theoretical configurations in Table 4 reveals that the observed ratios are more in line with RAS configurations than with NE.

We also find that subjects on average earn more from playing $D$ than from playing $H$ (ninth and tenth columns), which applies to all groups of all treatments. ${ }^{27}$

On average, groups earn $66.7 \%$ of the maximum sum of payoffs (last column of Table 5). In the groups of HD30 and HD50 the degrees of efficiency, i.e. the percentages of the maximum sum of payoffs, are approximately on the same level, while all these groups show a higher degree than each group in HD70. ${ }^{28}$ A likely explanation for the lower efficiency level in HD70 is that a link between two $D$ s, which increases efficiency (because $2 \cdot 60>70$ ), is an unfavorable link with a negative payoff $(60-70<0)$.

Result 1. In groups, more links are changed than actions. Moreover, $D$ is played more often than $H$, the $D$ action is kept longer than the $H$ action, and higher payoffs are earned by playing $D$ than by playing $H$. Groups reach a higher degree of efficiency in HD30 and HD50 than in HDY0.

\subsection{Analysis of Observed Decisions and Strategy Configurations}

In this section, we examine the consistency of the observed behavior with ER and with the behavioral hypotheses BR and ABR as well as the consistency of the observed configurations with NE and RAS (see Section 4). With these analyses, we also aim for a better understanding of Result 1. In particular, we ask: First, Do subjects react to changes according to ER? Second, How often are strategy changes in line with the BR or with the ABR? And how often is the (according to the ABR) expected payoff increase realized? Third, What is the total time in NE and in RAS configurations? How long do groups keep certain NE and RAS configurations?

We restrict our analyses in 5.2.1 and 5.2.2 to strategy changes that involve an action change: As argued on page 12, although ER and the ABR are defined for all types of strategy changes (see Section 4), strategy changes that involve an action change are better suited for our analyses than strategy changes that consist only of link changes.

\subsubsection{Consistency of Subjects' Reactions with ER}

After an action change, ER stipulate that the other group members will react by specific link adaptations (Definition 4). Do we observe ER-consistent behavior? We address this question by analyzing the other group members' behavior after an action change. That is, whenever a subject $i$ changes her action, we record the other five subjects' behavior if their first decision after $i$ 's change is to establish or delete a link to $i$, or if it is to change the action, provided the subject and $i$ are linked, or if they do not

\footnotetext{
${ }^{27}$ Sign test (pooled data of all 18 groups) $: p$-value $<0.05$.

${ }^{28}$ Comparison of degree of efficiency: Overall comparison (Kruskal-Wallis test): test statistic $=11.802$, degrees of freedom $=2, p$-value $<0.05$. Pairwise multiple comparison (Tukey Test): HD30 vs. HD50: $p$-value $>0.05$; HD30 vs. HD70: $p$-value $<0.05$; HD50 vs. HD70: $p$-value $<0.05$.
} 
Table 6: Other Group Members' Behavior After a Subject Changes her Action

\begin{tabular}{|c|c|c|c|c|}
\hline \multirow{4}{*}{ Treat. } & \multicolumn{3}{|c|}{ Other subjects' behavior after an action change } & \multirow{4}{*}{$\begin{array}{c}\text { Overall } \\
\text { consistency } \\
\text { with ER }\end{array}$} \\
\hline & Action change & Link change & No reaction & \\
\hline & Avg. proportion & Avg. proportion & Avg. proportion & \\
\hline & (consistent with ER) & (consistent with ER) & (consistent with ER) & \\
\hline HD30 & $8.8 \% \quad(0 \%)$ & $41.5 \% \quad(83.2 \%)$ & $49.7 \% \quad(92.4 \%)$ & $80.3 \%$ \\
\hline HD50 & $6.2 \% \quad(0 \%)$ & $43.8 \% \quad(82.1 \%)$ & $50.0 \% \quad(90.1 \%)$ & $80.8 \%$ \\
\hline HD70 & $2.1 \% \quad(0 \%)$ & $28.7 \% \quad(80.3 \%)$ & $69.3 \% \quad(97.1 \%)$ & $90.1 \%$ \\
\hline Avg. & $5.7 \% \quad(0 \%)$ & $38.0 \% \quad(81.9 \%)$ & $56.3 \% \quad(93.2 \%)$ & $83.7 \%$ \\
\hline
\end{tabular}

react. ${ }^{29}$ Most of our recorded changes occur during the first ten seconds after an action change: about $75 \%$ of all recorded link changes in all treatments, and about $50 \%$ in HD30 and HD50 and 74\% in HD70 of all recorded action changes.

After an action change, on average $86.9 \%$ of the other subjects' reactions by changes are link changes (i.e. they establish or delete a link to the considered subject) and only $13.1 \%$ of their first changes are action changes (this can be derived from Table 6). ${ }^{30}$ This high number of link changes compared with action changes supports the ER hypothesis of link reactions and the theoretical arguments in Section $4 .{ }^{31}$ If we take subjects that do not react into account, the percentage of action changes decreases to $5.7 \%$.

According to Table 6 , in $81.9 \%$ of the cases where a subject changes her link and in $93.2 \%$ of the cases where a subject does not react, the decision is in line with ER. ${ }^{32}$ Recorded action changes are of course never in line with ER. Overall, $83.7 \%$ of recorded behavior after an action change is consistent with ER. This high level of consistency supports the ER hypothesis.

\footnotetext{
${ }^{29}$ After $i$ 's action change, the recording of another subject $j$ 's behavior starts after a reaction time of one second and stops if $j$ makes a change, or if $i$ changes her action again or changes her link to $j$, or if the game ends. Here, $j$ 's action or link change is only counted if it occurs within 30 seconds after $i$ 's action change. Subject $j$ is counted as not reacting if $j$ does not change her action or her link to $i$ for at least ten seconds after $i$ 's change and if then the recording stops because $i$ changes her action again or changes her link to $j$, or the game ends, which may be later than 30s after $i$ 's initial action change.

${ }^{30}$ In all 18 groups we observe more first link changes than first action changes. Sign test (pooled data of all 18 groups): $p$-value $<0.05$.

${ }^{31}$ Note the difference from the argument in Section 5.1 on the relation of the numbers of link changes and action changes: now, every opponent may either change her link to the considered subject or change her action (or is not counted as reacting).

${ }^{32}$ For the analysis of the link changes, all possible cases are taken into consideration. First, the actionchanging subject $(i)$ and another subject $(j)$ are not linked: this case is counted when $j$ establishes a link to $i$, which is either consistent or inconsistent with ER. Second, there exists a single link established by $i$ to $j$ : this case is counted when $j$ establishes a link to $i$, which always contradicts ER. Third, there exists a single link established by $j$ to $i$ : this case is counted when $j$ deletes the link, which is either consistent with ER or not. Fourth, $i$ and $j$ have a double link: this case is counted when $j$ deletes the link, which is either consistent with ER or not (if $j$ is an $H$ and $i$ is a $D$ ).
} 
Result 2. There is evidence that subjects preferably react to action changes in line with Expected Linking Reactions (ER).

The lowest level of ER-consistent link changes is $64.1 \%$ in group 6 in HD70. In this group, we observe a phenomenon, which we call circular sponsoring, that is not captured by our theoretical criteria (see Section 5.3).

\subsubsection{Consistency of Subjects' Action Changes with the BR and the ABR}

Now the question arises how far subjects take these link reactions into consideration when deciding on their strategy. According to the BR, a player only considers her payoff that is directly induced by her strategy change (direct payoff effect) and does not incorporate other players' reactions, whereas the ABR assumes that a player does not take account of the direct payoff effect but considers her payoff after expected link adjustments of the other players (indirect payoff effect). Hence, to test consistency with the BR, we first examine subjects' payoff changes that are directly induced by their action change. Also, we examine how their payoff would have changed if the actionchanging subject also adjusted her links optimally (hypothetical direct payoff effect). For the ABR, we have to consider the development after a strategy change. Here, we first compute how the payoff of the action-changing subject would be affected if other group members adjusted their links according to ER and the player herself optimally adapted her links to these changes (hypothetical indirect payoff effect). Then we explore what actually happens after an action change by analyzing the following events and the actual payoff development of the action-changing subjects (actual indirect payoff effect).

Test of the BR. With respect to the BR we compute the direct payoff effect of action changes (i.e. a subject's payoff change only caused by her action change) and observe a clear picture in HD30 and HD50 (upper part of Table 7). ${ }^{33}$ Most changes from $H$ to $D$ lead to a payoff loss, while the majority of changes from $D$ to $H$ lead to a payoff increase. In HD70, the high percentage of cases in which a subject's payoff is not affected by her action change is due to the fact that the subjects are often unlinked. We find that in all six groups in all three treatments, a change from $H$ to $D$ leads more often to a weak payoff decrease than to a strict increase. ${ }^{34}$ Almost the opposite result holds for changes from $D$ to $H$ for HD30 and HD50: in five and six groups, the change from $D$ to $H$ induces more strict payoff increases than weak decreases and in one group the number of strict increases equals that of weak decreases. HD70 is an exception because

\footnotetext{
${ }^{33}$ Concretely, the values in Table 7 are the averages over the groups' percentages. These are the percentages of changes with positive direct payoff effect, no payoff effect, and negative direct payoff effect in the group, i.e. the percentages of changes where the payoff after the action change is higher than, equal to, and lower than the payoff before the change, respectively.

${ }^{34}$ Sign test (pooled data of all 18 groups): $p$-value $<0.05$.
} 
Table 7: Direct Payoff Effect of Strategy Changes (Test of the BR)

\begin{tabular}{ccccccc}
\hline & \multicolumn{5}{c}{ Direct payoff effect of action changes } \\
Treat. & Increase & Equal & Decrease & Increase & Equal & Decrease \\
\hline HD30 & $7.4 \%$ & $13.5 \%$ & $79.1 \%$ & $74.6 \%$ & $9.0 \%$ & $16.4 \%$ \\
HD50 & $7.4 \%$ & $7.9 \%$ & $84.7 \%$ & $66.1 \%$ & $16.3 \%$ & $17.6 \%$ \\
HD70 & $10.2 \%$ & $54.8 \%$ & $35.0 \%$ & $26.8 \%$ & $31.6 \%$ & $41.6 \%$ \\
\hline Avg. & $8.3 \%$ & $25.4 \%$ & $66.3 \%$ & $55.8 \%$ & $19.0 \%$ & $25.2 \%$ \\
\hline Hypothetical direct payoff effect of action changes with own optimal link adjustments & \multicolumn{4}{c}{$\boldsymbol{D}$ to $\boldsymbol{H}$} \\
Treat. & Increase & Equal & Decrease & Increase & Equal & Decrease \\
\hline HD30 & $42.3 \%$ & $2.4 \%$ & $55.3 \%$ & $89.5 \%$ & $2.6 \%$ & $7.9 \%$ \\
HD50 & $19.4 \%$ & $6.6 \%$ & $74.0 \%$ & $91.4 \%$ & $3.8 \%$ & $4.8 \%$ \\
HD70 & $20.5 \%$ & $52.0 \%$ & $27.5 \%$ & $92.0 \%$ & $2.7 \%$ & $5.3 \%$ \\
\hline Avg. & $27.4 \%$ & $20.4 \%$ & $52.2 \%$ & $91.0 \%$ & $3.0 \%$ & $6.0 \%$ \\
\hline
\end{tabular}

in five groups the majority of changes from $D$ to $H$ result in a weak loss for the subject. Pooling all groups, we do not get a significant result for changes from $D$ to $H .{ }^{35}$

A strategy change may also include some link changes that the subject plans to carry out after her action change. ${ }^{36}$ Since these link changes may interfere with other group members' reactions, we additionally compute how a subject's payoff would hypothetically be affected if she optimally adapted her links to her action change, while the other group members retained their current strategy (lower part of Table 7). The results for changes from $D$ to $H$ become clearer: in $91 \%$ of the cases, a change from $D$ to $H$ leads to a strict payoff increase for the subject. Also, in all groups of HD70 we do now observe a majority of payoff increases. ${ }^{37}$ Thus, it is likely that subjects that switch from $D$ to $H$ in HD70 plan to establish unambiguous links to $D$ s afterwards. On the other hand, even if we assume that subjects ceteris paribus optimally adjust their links to their change from $H$ to $D$, their payoff would still weakly decrease in most cases. ${ }^{38}$ We conclude that, if we take own BR-link adjustments after an action change into account, action changes from $D$ to $H$ are in line with the BR, while most decisions on changes from $H$ to $D$ contradict this rule.

\footnotetext{
${ }^{35}$ Sign test (pooled data of all 18 groups): $p$-value $>0.05$, with heterogeneous results across the treatments.

${ }^{36}$ Remember, a subject can change only one component of her strategy vector at any time.

${ }^{37}$ Sign test (pooled data of all 18 groups): in all 18 groups we observe a majority of strict payoff increases, $p$-value $<0.05$.

${ }^{38}$ Sign test (pooled data of all 18 groups): one group in HD30 with a majority of strict payoff increases and 16 groups with a majority of weak decreases, $p$-value $<0.05$. In one group in HD30 we observe an equal number of strict payoff increases and weak decreases.
} 
Table 8: Indirect Payoff Effect of Strategy Changes (Test of the ABR)

\begin{tabular}{ccccccc}
\hline \multicolumn{7}{c}{ Hypothetical indirect payoff effect of action changes } \\
Treat. & Increase & Equal & Decrease & Increase & Equal & Decrease \\
\hline HD30 & $82.8 \%$ & $2.7 \%$ & $14.5 \%$ & $39.4 \%$ & $0.0 \%$ & $60.6 \%$ \\
HD50 & $63.9 \%$ & $4.1 \%$ & $32.0 \%$ & $36.1 \%$ & $4.4 \%$ & $59.5 \%$ \\
HD70 & $81.9 \%$ & $8.6 \%$ & $9.5 \%$ & $46.0 \%$ & $29.5 \%$ & $24.5 \%$ \\
\hline Avg. & $76.2 \%$ & $5.2 \%$ & $18.7 \%$ & $40.5 \%$ & $11.3 \%$ & $48.2 \%$ \\
\hline \multicolumn{7}{c}{ Actual indirect payoff effect of action changes } \\
Treat. & Increase & Equal & Decrease & Increase & Equal & Decrease \\
\hline HD30 & $57.1 \%$ & $6.8 \%$ & $36.1 \%$ & $35.8 \%$ & $10.1 \%$ & $54.1 \%$ \\
HD50 & $67.4 \%$ & $3.6 \%$ & $29.1 \%$ & $35.3 \%$ & $7.0 \%$ & $57.7 \%$ \\
HD70 & $76.2 \%$ & $9.9 \%$ & $13.9 \%$ & $37.3 \%$ & $22.2 \%$ & $40.5 \%$ \\
\hline Avg. & $66.9 \%$ & $6.8 \%$ & $26.3 \%$ & $36.1 \%$ & $13.1 \%$ & $50.8 \%$ \\
\hline
\end{tabular}

Test of the $A B R$. We now consider the indirect payoff effect of action-changing subjects, i.e. we compare a subject's payoff before her action change with her payoff after the other group members and the subject herself adapt their links to her action change. First, we ask whether strategy changes that involve action changes are in line with the ABR. To answer this question, we examine how the payoff of an action-changing subject would hypothetically be affected if the other group members adapted their links to the action change according to ER and the subject herself optimally adjusted her links to her action change and the others' ER-consistent link reactions. The hypothetical payoff changes in the upper part of Table 8 present a picture that is contrary to the respective result of the direct payoff effects in Table 7 . In particular, changes from $H$ to $D$ are profitable in most cases ${ }^{39}$ while changes from $D$ to $H$ are not. ${ }^{40}$

Second, we analyze whether actual play confirms the hypothetical payoffs. We compute the actual indirect payoff effect, i.e. a subject's actual payoff change after all group members adapt their links to her action change. For this purpose, we examine the sequence of link changes after an action change until the next action change in the group occurs, because action changes essentially alter the strategic situation and are not part of ER. The indirect payoff effect is computed as the sign of the difference between a

\footnotetext{
${ }^{39}$ Sign test (pooled data of all 18 groups): 15 groups with a majority of strict payoff increases and two groups in HD50 with a majority of weak payoff decreases, $p$-value $<0.05$. In one group of HD30 we observe an equal number of strict payoff increases and decreases.

${ }^{40} \mathrm{Sign}$ test (pooled data of all 18 groups): three groups (one in HD30 and two in HD70) with a majority of strict payoff increases and 14 groups (five in HD30, six in HD50, and three in HD70) with a majority of weak payoff decreases, $p$-value $<0.05$. In one group in HD70 we observe an equal number of strict payoff increases and unchanged (equal) payoffs.
} 
subject's payoff at the end of the sequence and her payoff right before her action change. The picture is similar to the hypothetical effect (lower part of Table 8): the majority of changes from $H$ to $D$ result in a payoff increase, while the majority of changes from $D$ to $H$ lead to a payoff decrease. ${ }^{41}$

From observing similar actual and hypothetical indirect payoff effects on average, and from additional support by Result 2, we infer that the observed payoff developments are mainly caused by ER-consistent link reactions.

Moreover, the fact that most changes from $H$ to $D(D$ to $H)$ induce a payoff increase (decrease) for the subjects during the course of link adaptations after their action change serves as a plausible explanation why the subjects on average keep the $H$ action shorter than the $D$ action (Result 1 ). Note that because link reactions lag behind action changes, subjects usually benefit from a switch from $D$ to $H$ for a short span of time, ${ }^{42}$ which might induce the observed BR-behavior of changing from $D$ to $H$.

Recapitulating, from the analysis of the payoff effects we conclude that most changes from $H$ to $D$ clearly contradict the BR, while they support the ABR. On the other hand, most changes from $D$ to $H$ are consistent with the BR and contradict the ABR. In summarizing the analyses in this section we draw the following conclusion.

Result 3. Most action changes from $D$ to $H$ are consistent with the $B R$ if the action changing player optimally (according to BR) adapts her links to her action change, but contradict the ABR. Action changes from $H$ to $D$ are in accord with the $A B R$ but contradict the BR.

\subsubsection{Consistency of Observed Strategy Configurations with $N E$ and $R A S$}

Now we check whether the observed strategy configurations are RAS or NE. As an indicator we measure the amount of time groups spend in RAS and NE (see Table 9). Because each subject has five links to decide on and a lot of deviations from the predicted network are possible, we additionally allow for link errors in our analysis. In Table 9, the column for zero link errors displays the results when we do not allow for any deviation from the respective network. In the next columns we allow for one, two, and three link errors in the network. An error is registered whenever a link deviates from the network prescribed by the respective criterion, that is, a link that should not be established as well as a missing link. ${ }^{43}$ To get an impression of the number of RAS and

\footnotetext{
${ }^{41}$ Changes from $H$ to $D$ result in a majority of strict payoff increases in 14 groups and in a majority of weak decreases in four groups (two in HD30 and two in HD50). Sign test (pooled data of all 18 groups): $p$-value $<0.05$. Changes from $D$ to $H$ result in a majority of weak payoff decreases in 14 groups and in a majority of strict payoff increases in two groups (one in HD30 and one in HD70). Sign test (pooled data of all 18 groups): $p$-value $<0.05$. In one group in HD50 and in one group in HD70 we observe an equal number of strict payoff increases and weak payoff decreases.

${ }^{42}$ This span of time is on average 12.3 $\mathrm{s}$ in HD30, 8.5 $\mathrm{s}$ in HD50, and $34.7 \mathrm{~s}$ in HD70.

${ }^{43} \mathrm{~A}$ double link is registered as one error if the link should be there and as two errors if no link should be there. In case of a single link of a $D$ to an $H$ we register two errors because $D$ has an erroneous link
} 
Table 9: Percentage of Time Spent in NE and RAS Configurations, Avg. Dwell Time in Seconds, and Avg. Starting Time in Seconds

\begin{tabular}{|c|c|c|c|c|c|c|c|c|c|}
\hline \multirow[t]{3}{*}{ Treat. } & \multicolumn{3}{|c|}{ Percentage of time } & & \multicolumn{4}{|c|}{ Avg. dwell time [s] } & $\begin{array}{l}\text { Avg. starting } \\
\quad \text { time }[\mathrm{s}]\end{array}$ \\
\hline & \multicolumn{4}{|c|}{ Number of link errors } & \multicolumn{4}{|c|}{ Number of link errors } & \\
\hline & 0 & 1 & 2 & 3 & 0 & 1 & 2 & 3 & \\
\hline & \multicolumn{8}{|c|}{$\mathrm{NE}$} & \\
\hline HD30 & 4.0 & 11.0 & 17.7 & 22.2 & 5.7 & 9.6 & 13.7 & 22.0 & 986 \\
\hline HD 50 & 2.1 & 4.6 & 7.7 & 10.5 & 10.5 & 15.6 & 20.6 & 23.5 & 594 \\
\hline HD70 & 15.8 & 27.5 & 33.8 & 39.5 & 24.6 & 32.5 & 43.2 & 45.7 & 861 \\
\hline \multirow[t]{2}{*}{ Avg. } & 7.3 & 14.4 & 19.7 & 24.0 & 13.6 & 19.2 & 25.8 & 30.4 & 803 \\
\hline & \multicolumn{8}{|c|}{ RAS } & \\
\hline HD30 & 12.3 & 26.3 & 40.5 & 49.7 & 14.1 & 21.0 & 29.0 & 33.7 & 1205 \\
\hline HD 50 & 6.4 & 21.1 & 36.0 & 49.2 & 6.7 & 12.9 & 19.0 & 23.4 & 1014 \\
\hline HD70 & 26.7 & 46.6 & 57.2 & 65.3 & 17.3 & 23.1 & 31.5 & 35.2 & 1040 \\
\hline Avg. & 15.1 & 31.4 & 44.6 & 54.8 & 12.7 & 19.0 & 26.5 & 30.8 & 1087 \\
\hline
\end{tabular}

NE configurations, note that of $2^{36}$ possible different strategy configurations, in HD30 fewer than 7104 are RAS and fewer than 62560 are NE. ${ }^{44}$

We observe that for all considered numbers of link errors, RAS captures a larger part of the observed configurations than NE does. ${ }^{45}$ The time subjects spend in RAS configurations may appear low, but it strongly increases if we allow for link errors.

Next, we examine if behavior stabilizes in NE or RAS configurations. First, we find significantly higher dwell times in NE or RAS configurations with zero link errors (sixth column of Table 9) than in all other configurations, which are neither NE nor RAS. ${ }^{46}$

and $H$ has a missing link. Hence, our approach of counting link errors is very meticulous.

${ }^{44}$ These numbers are calculated without considering the additional restrictions on the numbers of links of a $D$ (Table A.14). There exist less than 89872 RAS configurations and 197 NE in HD50 as well as 21 RAS configurations and $57 \mathrm{NE}$ in HD70.

${ }^{45} 0$ link errors (le): 14 groups stay longer in RAS configurations than in NE, while in three groups (two in HD30, one in HD50) it is the other way round, and in one group in HD70 the two coincide. Sign test (pooled data of all 18 groups): $p$-value $<0.05$.

1 le: 16 groups stay longer in RAS configurations than in NE, while in two groups in HD30 it is the other way around. Sign test (pooled data of all 18 groups): $p$-value $<0.05$.

2 and 3 le: 17 groups stay longer in RAS configurations than in NE, while in one group in HD30 it is the other way around. Sign test (pooled data of all 18 groups): $p$-value $<0.05$.

${ }^{46}$ The dwell times of the other configurations are calculated as $1800 \mathrm{~s}$ minus the total time in RAS configurations and in NE, divided by the number of observed networks that are not RAS or NE.

RAS: 16 groups have longer dwell times in RAS than in non-RAS and non-NE configurations, while in two groups (one in HD50, one in HD70) it is the other way around. Sign test (pooled data of all 18 groups): $p$-value $<0.05$.

NE: 14 (of 17, one in HD30 did not reach any NE) groups have longer dwell times in NE than in 
The average dwell time in these configurations is $3.8 \mathrm{~s}$ in HD30, $3.5 \mathrm{~s}$ in HD50, and $3.4 \mathrm{~s}$ in HD70. These data are not shown in Table 9. We also compute the dwell times for NE and RAS configurations with one, two, and three link errors. The dwell time of a certain configuration starts when the group reaches this configuration up to the respective number of link errors and stops when the number of link errors exceeds the threshold or an action change occurs. In order to be counted, the exact configuration has to be reached at least once. From the dwell times in Table 9 and the prior comparison with other configurations we conclude that behavior stabilizes both in NE and in RAS, but not for very long. ${ }^{47}$ This issue will be addressed in the next Section 5.3.

We also determine the time when RAS and NE configurations are played in the 30 minutes (1800 seconds) period and observe that RAS configurations are on average played later than NE configurations (last column of Table 9). ${ }^{48}$ This might indicate that the subjects learn from experiences earlier in the game that the others preferentially react according to ER. Also, note that groups play RAS configurations more often than NE. This can be seen from their similar stability (dwell times) but higher percentage of time in RAS configurations (see also Tables 9 and 10 in the online supplementary material).

The following result summarizes our findings of this section.

Result 4. RAS configurations and their direct neighborhood of a few link deviations are more prevalent than NE, in terms of time and frequency. NE and $R A S$ configurations are more stable than other configurations are on average. RAS configurations are on average played later than NE configurations.

\subsection{Instability and Other Patterns of Behavior}

Investigating ER, the ABR, the BR, and RAS enabled us to understand the main patterns of behavior in the experiment. However, these criteria of course abstract from many other and more diverse behavior patterns. For this reason, we now examine the cases where groups leave NE and RAS configurations and investigate strategy changes that are not in line with the BR and the ABR. Whenever a NE or a RAS configuration is left, the change is not in line with $\mathrm{BR}$ or $\mathrm{ABR}$, respectively. Table 10 displays the relative frequency of all types of strategy changes that can terminate a NE or RAS. We see that frequencies in HD30 and HD50 are similar but HD70 differs.

In HD30 and HD50, NE are mainly terminated because an $H$ changes to $D(H a)$ or because a $D$ deletes a link $(D l-)$. The former might be ABR-conform behavior as illustrated in the example in Section 4: the switching player anticipates that the $H$ s will

non-RAS and non-NE configurations, while in three groups (one in HD30, one in HD50, one in HD70) it is the other way around. Sign test (pooled data of all 18 groups): $p$-value $<0.05$.

${ }^{47}$ We do not find significant differences between the dwell times in NE and RAS configurations.

${ }^{48}$ In 14 (of 16) groups RAS configurations start later than NE on average, in two groups it is the other way round. (In HD30 one group never reached a NE and in one group in HD70 RAS and NE coincide.) Sign test (pooled data of all 18 groups): $p$-value $<0.05$. 
Table 10: Deviations from NE and RAS Configurations

\begin{tabular}{ccrrrrrr}
\hline Treat. & \multicolumn{7}{c}{ Relative frequency of types of deviation [\%] } \\
\cline { 2 - 7 } & $D l-$ & $H l-$ & $D l+$ & $H l+$ & $D a$ & $H a$ & Sum \\
\cline { 2 - 7 } HD30 & 38.2 & 1.8 & 5.5 & 3.6 & 9.1 & 41.8 & 100 \\
HD50 & 36.8 & 5.3 & 5.3 & 10.5 & 5.3 & 36.8 & 100 \\
HD70 & - & 51.5 & 28.3 & 7.1 & 4.0 & 9.1 & 100 \\
\hline & \multicolumn{7}{c}{ RAS } \\
HD30 & 52.3 & 10.5 & 2.3 & 0.0 & 29.1 & 5.8 & 100 \\
HD50 & 67.0 & 4.4 & 6.6 & 2.2 & 13.2 & 6.6 & 100 \\
HD70 & - & 31.5 & 43.5 & 4.2 & 16.1 & 4.8 & 100 \\
\hline
\end{tabular}

Types of deviations:

$D$ deletes a (single) link $(D l-), H$ deletes a (single) link $(H l-), D$ establishes a (double) link $(D l+)$, $H$ establishes a (double) link $(H l+), D$ changes to $H(D a), H$ changes to $D(H a)$.

establish links to her, thereby making her switch profitable. The motivation for a $D$ who deletes her link to another $D$ appears clear: the $D$ wants the other $D$ to take over the ambiguous link. ${ }^{49}$ Note that this is not only the most frequent case of terminating RAS configurations in HD30 (52.3\%) and HD50 (67\%), it is even the most frequently observed behavior in HD30 and HD50 not consistent with ER. The second most frequent case of leaving a RAS configuration in HD30 and HD50 is the switch from $D$ to $H(D a)$. As pointed out in Section 5.2.2, changes from $D$ to $H$ often seem to be driven by the attempt to make short-term profits, pointing to the relevance of the BR.

In HD70, established links by $D(D+)$ and deleted links by $H(H-)$ are mainly responsible for terminating both $\mathrm{NE}$ and RAS configurations. In this treatment, the frequency and duration of NE and RAS is relatively high. So, the deviations may point at additional attempts to earn money, which take the time dimension into account. The incentive for a $D$ to establish a link to another $D$, although her payoff from this link becomes negative, will be discussed in the next paragraph. A closer look at the $\mathrm{Hs}$ who delete their links to the $D \mathrm{~s}(H l-)$ reveals that this decision is mostly followed by a change to $D$, where the subject obviously expects the $H_{\mathrm{s}}$ to establish links to her.

In HD70, the opportunities to earn money are sparse since only a link from $H$ to $D$ provides both players with a positive payoff. Contrary to HD30 and HD50, for a $D$ it is not profitable to establish a link to another $D$. However, there is a way for $D$ s not only to increase their collective payoff but also their individual payoffs by what we call circular sponsoring: three (or more) Ds are linked such that every $D$ pays for one link.

\footnotetext{
${ }^{49}$ Note, only in the NE of HD30 a $D$ may also establish a link to $H$. So, $D$ - for NE of HD30 also includes the deletion of a link of a $D$ to an $H$.
} 
In this way, the payoff of each $D$ increases by 50 ExCU per minute. In groups 1,2 , and 6 in HD70, we observe circular sponsoring. Especially in group 6, where the criteria that we are investigating do not fit the data well, the occurrence of triadic circles in more than $30 \%$ of the time supports sponsoring as an alternative explanation.

\section{Conclusion}

Our theoretical and experimental analyses provide new insights into the coevolution of actions and networks.

Coming back to the questions posed in the introduction, neither the network nor the action configuration remains stable. Instead, subjects make use of links and actions to influence their payoff. Thus, if network and actions are equally flexible (e.g., because both can be adjusted whenever the player wants) the game cannot be approximated by models of action choice in fixed networks or pure network formation models.

The experimental data support the central assumption of ER, namely that subjects adapt to new strategy configurations by adjusting links rather than by adjusting actions (see Result 2). The underlying assumption is that the direct consequences of a link change, which impacts only one bilateral interaction, are easier to assess than those of an action change. Furthermore, there seem to exist norms on who has to pay for the links (as included in ER, see Result 2). In particular, subjects who change from $H$ to $D$ apparently take these norms into account (Result 3). Strategy configurations that do not allow for profitable unilateral deviations under these norms are observed to last a relatively long time, that is, RAS configurations are observed for a longer time than NE (Result 4). Stabilization on a low level is observed for both criteria (Result 4). In HD30 and HD50, stability is most often disturbed by a D that deletes the ambiguous link to another D in order to induce her to take over the link, which can be interpreted as payoff-equalizing behavior. Aside from this disagreement about who should pay for ambiguous links, myopic (BR) and farsighted (ABR) adaptations seem to be to a large extent responsible for the low level of stability of RAS and NE, respectively.

Although $D$ s are tempted to deviate to $H$ to collect a higher profit by exploiting $D$ s (Result 3), this behavior is not profitable in the long run and the payoffs from playing $D$ are higher than those from playing $H$ (Result 1 ). Thus, amicable behavior (as $D$ ) pays in the long run. This is in line with the finding of Tinsley et al. (2002) that a reputation as a tough bargainer may have negative effects on payoffs. Whereas their result is due to the lower value created by negotiation (e.g. because information is retained), in our model the reason for the lower payoffs is that tough bargainers are avoided by others, have to bear the cost to set up the negotiation, and that their presence causes efficiency losses. In our model, amicability is not self-enforcing, but may nevertheless fare better than exploitation. The credible threats, which can immediately be played out, increase the level of amicable behavior above the level in NE.

The theoretical criteria that we propose (ABR and RAS) take into account the norms on who bears the link cost (ER). From certain points of view, they capture our 
experimental observations better than the more traditional $\mathrm{BR}$ and $\mathrm{NE}$ alternatives considered. An exception is the switch from $D$ to $H$, which is better in line with BR. These insights could spur the development of new solution concepts for continuous games involving strategic networking.

Running the experiment in continuous time is well suited to our investigation. This approach allows subjects to make immediate adaptations but minimizes the risk of coordination failure due to simultaneous changes. We believe that the continuous time setting is a crucial factor for sustaining amicability. This view is further confirmed by the high rates of cooperation in an experimental study on the prisoner's dilemma in continuous time (Friedman and Oprea, 2009).

A possible direction for further research might be to test the robustness of our results by analyzing network formation with other pairwise games or to extend the scope to related games like buyer-seller games in endogenous bipartite networks.

\section{Acknowledgements}

Financial support from the Deutsche Forschungsgemeinschaft, SFB 504, at the University of Mannheim, is gratefully acknowledged. We thank Bodo Vogt for helpful comments on an earlier version. The suggestions of an anonymous referee and the editor greatly helped to improve the paper.

\section{References}

Bala, V., Goyal, S., 2000. A noncooperative model of network formation. Econometrica $68,1181-1229$.

Berninghaus, S. K., Vogt, B., 2006. Network formation in symmetric $2 \times 2$ games. Homo Oeconomicus 23, 421-466.

Bramoullé, Y., López-Pintado, D., Goyal, S., Vega-Redondo, F., 2004. Network formation and anti-coordination games. Int. J. Game Theory 33, 1-19.

Corbae, D., Duffy, J., 2008. Experiments with network formation. Games Econ. Behav. 64 (1), 81-120.

Cornell, B., Roll, R., 1981. Strategies for pairwise competitions in markets and organizations. Bell J. Econ. 12 (1), 201-213.

Corten, R., Buskens, V., 2010. Co-evolution of conventions and networks: An experimental study. Social Networks 32 (1), 4-15.

Ehrhart, K.-M., 1997. Kollektivgutspiele in stetiger und diskreter Zeit. Ph.D. thesis, University of Karlsruhe. 
Friedman, D., Oprea, R., 2009. A continuous dilemma. Working paper, University of California, Santa Cruz.

Friedman, J. W., Mezzetti, C., 2001. Learning in games by random sampling. J. Econ. Theory $98,55-84$.

Hojman, D. A., Szeidl, A., 2006. Endogenous networks, social games, and evolution. Games Econ. Behav. 55, 112-130.

Jackson, M. O., Watts, A., 2002. On the formation of interaction networks in social coordination games. Games Econ. Behav. 41, 265-291.

Jackson, M. O., Watts, A., 2008. Equilibrium existence in bipartite social games: A generalization of stable matchings. Econ. Bull. 3 (12), 1-8.

Jackson, M. O., Watts, A., 2010. Social games: Matching and the play of finitely repeated games. Games Econ. Behav., 70 (1), 170-191.

Jackson, M. O., Wolinsky, A., 1996. A strategic model of social and economic networks. J. Econ. Theory 71, 44-74.

Jehiel, P., 2001. Limited foresight may force cooperation. Rev. Econ. Stud. 68, 369-391.

Kosfeld, M., 2004. Economic networks in the laboratory: A survey. Review of Network Economics 3, 20-41.

Lipman, B. L., 1986. Cooperation among egoists in Prisoners' Dilemma and Chicken games. Public Choice 51, 315-331.

Maynard Smith, J., 1982. Evolution and the Theory of Games. Cambridge: Cambridge University Press.

Morris, S., 1995. The common prior assumption in economic theory. Econ. Philos. 11, $227-253$.

Neugebauer, T., Poulsen, A., Schram, A., 2008. Fairness and reciprocity in the HawkDove game. J. Econ. Behav. Organ. 66 (2), 243-250.

Riedl, A., Ule, A., 2002. Exclusion and cooperation in social network experiments. Working paper, University of Amsterdam.

Simon, L. K., Stinchcombe, M. B., 1989. Extensive form games in continuous time: Pure strategies. Econometrica 57, 1171-1214.

Skyrms, B., Pemantle, R., 2000. A dynamic model of social network formation. Proceedings of the National Academy of Sciences of the USA 97, 9340-9346. 
Snyder, G. H., 1971. "Prisoner's Dilemma" and "Chicken" models in international politics. International Studies Quarterly 15, 66-103.

Tinsley, C. H., O’Connor, K. M., Sullivan, B. A., 2002. Tough guys finish last: the perils of a distributive reputation. Organizational Behavior and Human Decision Processes $88,621-642$.

Ule, A., 2005. Exclusion and cooperation in networks. Ph.D. thesis, Tinbergen Institute Research Series no. 354.

\section{Appendix A. NE and RAS Configurations}

Tables A.11 and A.12 show for all parameter combinations for $a>b>c>d>0$ and $k>0$ the properties of NE and RAS configurations, respectively. These conditions may also serve as a basis for a proof of existence. In all NE and RAS configurations no double links exist. Types of links that are not mentioned in the tables are not established. Additional restrictions on the numbers of links of players may occur. Then, $l^{x y}$ denotes the number of links from an $x$ to a $y, x, y \in\{H, D\}$. The cases in the first column in Table A.12 are described in Table B.15.

Table A.11: NE (Bramoullé et al., 2004; Berninghaus and Vogt, 2006)

\begin{tabular}{lll}
\hline & Number of $H \mathrm{~s}\left(n_{H}\right)$ & Link structure (single links) \\
\hline$d>k>0$ & $\frac{n(a-b)+c-d}{a-b+c-d} \geq n_{H} \geq \frac{(n-1)(a-b)}{a-b+c-d}$ & All players are linked. \\
$c>k>d$ & $\frac{n(a-b)+c-k}{a-b+c-k} \geq n_{H}$ & All $H \mathrm{~s}$ and $D$ s are linked via a link of $H$ or of $D$; all $D \mathrm{~s}$ \\
& $\geq \frac{(n-1)(a-b)+l^{D H}(k-d)}{a-b+c-d}$ & are linked to each other and $l^{D H} \leq \frac{\left(n-n_{H}-1\right)(b-a)+n_{H}(c-d)}{k-d}$ \\
$b>k>c$ & $n_{H} \geq \frac{(n-1)(a-b)}{a-b+c-d}$ & for all $D \mathrm{~s}$. \\
$a>k>b$ & $n_{H} \geq \frac{(n-1)(a-k)}{a-k+c-d}$ & $H$ s establish links to all $D \mathrm{~s} ;$ all $D \mathrm{~s}$ are linked to each other. \\
$k>a$ & $n_{H}$ arbitrary & Complete bipartite graph $(H \mathrm{~s}$ and $D$ s) with links of $H \mathrm{~s}$. \\
\hline
\end{tabular}

\section{Appendix B. Proof of the RAS Configurations of Table A.12}

According to the ABR, a player will establish missing ambiguous and unambiguous links and delete unfavorable and double ambiguous links. Thus, in a RAS configuration, all ambiguous and unambiguous links are established and no unfavorable or double links exist. Table B.15 gives an overview of the classification of links from $x$ to $y$ (denoted by $x y$ ) for $x, y \in\{H, D\}$ for the seven different conditions for the cost parameter $k$.

The inequalities in Table B.16 compare the payoff for each case and for $H$ and for $D$ in a situation where deviations in links alone are not profitable under the ABR (i.e. only favorable single links according to Table B.15 exist) with the payoff that this player expects if she changes her action and the others adapt their links according to ER. Only strategy configurations $s$ that fulfill these conditions are candidates for RAS 
Table A.12: RAS Configurations (the Cases in the First Column are Described in Table B.15)

\begin{tabular}{lll}
\hline Case & Number of $H \mathrm{~s}\left(n_{H}\right)$ & Link structure (single links) \\
\hline 1 & $\frac{n(a-b)+c-d-l^{H H} k}{a-b+c-d} \geq n_{H}$ & All players are linked; $H \mathrm{~s}$ establish links to all $D \mathrm{~s} ;$ restrictions \\
& $\geq \frac{(n-1)(a-b-k)+l^{D} k}{a-b+c-d-k}$ & on numbers of links $l^{H H}$ for all $H \mathrm{~s}$ and $l^{D D}$ for all $D \mathrm{~s}$ can be \\
2 & $\frac{n(a-b)+c-d}{a-b+c-d} \geq n_{H} \geq \frac{(n-1)(a-b)}{a-b+c-d}$ & derived from inequalities. \\
& $\begin{array}{l}\text { All players are linked; } H \mathrm{~s} \text { establish links to all } D \mathrm{~s} \text {; restrictions } \\
\text { on } l^{H H} \text { for all } H \mathrm{~s} \text { and } l^{D D} \text { for all } D \mathrm{~s} \text { apply. }\end{array}$ \\
3,5 & $\frac{n(a-b)+c}{a-b+c} \geq n_{H} \geq \frac{(n-1)(a-b-k)+l^{D D} k}{a-b+c-k}$ & $H \mathrm{~s}$ establish links to all $D \mathrm{~s} ; H \mathrm{~s}$ are not linked to $H \mathrm{~s}$; all $D \mathrm{~s}$ \\
4 & $\frac{n(a-b)+c-k}{a-b+c-k} \geq n_{H} \geq \frac{(n-1)(a-b)+l^{D H} k}{a-b+c}$ & are linked to each other; restrictions apply to $l^{D D}$ for all $D \mathrm{~s}$. \\
& No link between $H \mathrm{~s} ; H \mathrm{~s}$ establish links to $D \mathrm{~s} ; D \mathrm{~s}$ establish \\
6 & $\frac{n(a-k)+c}{a+c-k} \geq n_{H} \geq \frac{(n-1)(a-k)}{a+c-k}$ & links to $H \mathrm{~s}$ and to $D \mathrm{~s} ;$ restrictions apply to $l^{D H}$ for all $D \mathrm{~s}$. \\
7 & $n_{H}$ arbitrary & Complete bipartite graph (of $H \mathrm{~s}$ and $D \mathrm{~s})$ with links of $H \mathrm{~s}$ to \\
& Ds only. \\
\hline
\end{tabular}

Table A.13: NE of the HDN Game in the Experiment

\begin{tabular}{|c|c|c|}
\hline$k$ & $n_{H}: n_{D}$ & Link structure (single links) \\
\hline 30 & $3: 3^{a}, 4: 2$ & $\begin{array}{l}\text { All } H \mathrm{~s} \text { and } D \mathrm{~s} \text { are linked via a link of } H \text { or of } D \text {; } \\
\text { no link between } H \mathrm{~s} ; D \mathrm{~s} \text { are linked to each other. }\end{array}$ \\
\hline 50 & $3: 3,4: 2,5: 1,6: 0$ & $\begin{array}{l}H \mathrm{~s} \text { establish links to all } D \mathrm{~s} \text {; } \\
\text { no link between } H \mathrm{~s} ; D \mathrm{~s} \text { are linked to each other. }\end{array}$ \\
\hline 70 & $2: 4,3: 3,4: 2,5: 1,6: 0$ & Complete bipartite graph (of $H \mathrm{~s}$ and $D \mathrm{~s}$ ) with links of $H \mathrm{~s}$ to $D \mathrm{~s}$ only. \\
\hline
\end{tabular}

${ }^{a}$ For $k=30$, the action distribution $3 H: 3 D$ constitutes a NE only if no $D$ has more than two links to $H$ s. If every $D$ has exactly two links to $H$ s, then the NE is non-strict.

configurations. If these inequalities are valid for all $H$ s and for all $D$ s, no player deviates according to the ABR and thus the configuration is RAS. Table A.12 combines the inequalities and summarizes the results. 
Table A.14: RAS Configurations of the HDN Game in the Experiment

\begin{tabular}{|c|c|c|}
\hline$k$ & $n_{H}: n_{D}$ & Link structure (single links) \\
\hline 30 & $1: 5,2: 4^{b}$ & $\begin{array}{l}H \mathrm{~s} \text { establish links to all } D \mathrm{~s} ; \text { no link between } H_{\mathrm{s}} \\
D \mathrm{~s} \text { are linked to each other. }\end{array}$ \\
\hline 50 & $0: 6,1: 5,2: 4^{c}$ & $\begin{array}{l}H \mathrm{~s} \text { establish links to all } D \mathrm{~s} ; \text { no link between } H \mathrm{~s} \text {; } \\
D \mathrm{~s} \text { are linked to each other. }\end{array}$ \\
\hline 70 & $1: 5,2: 4$ & Complete bipartite graph (of $H \mathrm{~s}$ and $D \mathrm{~s}$ ) with links of $H \mathrm{~s}$ to $D \mathrm{~s}$ only. \\
\hline
\end{tabular}

${ }^{b}$ For $k=30$ and $n_{H}=1$, the additional condition restricts the number of links of each $D$ to $D$ s to $l^{D D}=2$ (see Table A.12). For $n_{H}=2$, the condition $l^{D D} \leq 3$ imposes no restriction.

${ }^{c}$ For $k=50$ and $n_{H}=0$ and $n_{H}=1$, the additional condition restricts the number of links of each $D$ to $D$ s to $l^{D D} \leq 3$ (see Table A.12). For $n_{H}=2$, the condition $l^{D D} \leq 3$ imposes no restriction.

Table B.15: Classification of Links as Unambiguous, Ambiguous, and Unfavorable

\begin{tabular}{lllll}
\hline Case & Description & Unambiguous & Ambiguous & Unfavorable \\
\hline 1 & $d \geq k>0$ and $a-k>c$ & $H D$ & $D D, H H$ & $D H$ \\
2 & $d \geq k>0$ and $a-k \leq c$ & - & all & - \\
3 & $c \geq k>d$ and $a-k>c$ & $H D$ & $D D$ & $H H, D H$ \\
4 & $c \geq k>d$ and $a-k \leq c$ & - & $H D, D D, D H$ & $H H$ \\
5 & $b \geq k>c$ & $H D$ & $D D$ & $H H, D H$ \\
6 & $a \geq k>b$ & $H D$ & - & $D D, H H, D H$ \\
7 & $k>a$ & - & - & all \\
\hline
\end{tabular}

Table B.16: Inequalities That Have to Hold in RAS Configurations for Every Player

\begin{tabular}{|c|c|c|}
\hline Case & $\begin{array}{l}\text { No } \\
\text { devi- } \\
\text { ation } \\
\text { by }\end{array}$ & Condition \\
\hline 1 & $\begin{array}{l}H \\
D\end{array}$ & $\begin{array}{l}\left(n-n_{H}\right) a+\left(n_{H}-1\right) d-\left(n-n_{H}+l^{H H}\right) k \geq\left(n-n_{H}\right) b+\left(n_{H}-1\right) c-\left(n-n_{H}\right) k \\
\left(n-n_{H}-1\right) b+n_{H} c-l^{D D} k \geq\left(n-n_{H}-1\right)(a-k)+n_{H} d\end{array}$ \\
\hline 2 & $\begin{array}{l}H \\
D\end{array}$ & $\begin{array}{l}\left(n-n_{H}\right) a+\left(n_{H}-1\right) d-\left(l^{H H}+l^{H D}\right) k \geq\left(n-n_{H}\right) b+\left(n_{H}-1\right) c-\left(l^{H H}+l^{H D}\right) k \\
\left(n-n_{H}-1\right) b+n_{H} c-\left(l^{D H}+l^{D D}\right) k \geq\left(n-n_{H}-1\right) a+n_{H} d-\left(l^{D H}+l^{D D}\right) k\end{array}$ \\
\hline 3,5 & $\begin{array}{l}H \\
D\end{array}$ & $\begin{array}{l}\left(n-n_{H}\right)(a-k) \geq\left(n-n_{H}\right)(b-k)+\left(n_{H}-1\right) c \\
\left(n-n_{H}-1\right) b+n_{H} c-l^{D D} k \geq\left(n-n_{H}-1\right)(a-k)\end{array}$ \\
\hline 4 & $\begin{array}{l}H \\
D\end{array}$ & $\begin{array}{l}\left(n-n_{H}\right) a-l^{H D} k \geq\left(n-n_{H}\right) b+\left(n_{H}-1\right) c-\left(l^{H D}+n_{H}-1\right) k \\
\left(n-n_{H}-1\right) b+n_{H} c-\left(l^{D D}+l^{D H}\right) k \geq\left(n-n_{H}-1\right) a-l^{D D} k\end{array}$ \\
\hline 6 & $\begin{array}{l}H \\
D\end{array}$ & $\begin{array}{l}\left(n-n_{H}\right)(a-k) \geq\left(n_{H}-1\right) c \\
n_{H} c \geq\left(n-n_{H}-1\right)(a-k)\end{array}$ \\
\hline 7 & $\begin{array}{l}H \\
D\end{array}$ & $\begin{array}{l}\text { no links are established, payoff is zero } \\
\text { no links are established, payoff is zero }\end{array}$ \\
\hline
\end{tabular}




\section{Appendix C. Translation of Experimental Instructions}

This appendix contains a translation of the instructions of the experiment. The original instructions are in German. The following instruction belongs to Treatment HD30. Deviations in Treatments HD50 and HD70 are given in squared brackets, where the first entry corresponds to HD50, and the second to Treatment HD70. In the three treatments, instructions differ with respect to costs for active links (30, 50, and 70) as well as conversion rates of 400, 300, and 170 ExCU per Euro, respectively.

\section{Instructions}

You are participating in an experiment on interactive decision making with several participants. In this experiment, you can earn cash. How much you earn, depends on your own decisions and the decisions of other participants. In the experiment, payoffs earned are measured in so-called experimental currency units [ExCU]. The sum of ExCU you earn in total will be transformed into Euro at the end of the experiment and paid out in cash. Each participant makes his decisions separate from the remaining participants sitting at his computer-terminal. Communication between participants is not allowed.

At the beginning of the experiment, you will be randomly matched with five other participants to form a group of six. The participants of a group are not necessarily sitting side by side. The composition of a group persists during the course of the experiment. There is no interaction with other groups. The six members of your group will be randomly assigned internal labels from one to six. However, each participant is denoted on his screen as player $\boldsymbol{T} \mathbf{1}$. In the following, the term participant will be used only to denote participants of your group. Any other participant in your group obtains exactly the same instructions as you do.

The experiment runs for $\mathbf{3 0}$ minutes. During this time you can build or delete links

to the other participants of your group. In addition, you have to choose between two alternative actions " $\mathrm{X}$ " and "Y", in short alternatives " $\mathrm{X}$ " and "Y." You obtain a continuous payoff stream whose size depends on your links and on the alternative you have chosen.

Links. There are two ways to be connected to other participants in your group.

You can decide to build links to other participants by yourself. These links will be called your active links. Your active links will be displayed as arrows pointing from you to other participants. You can build active links to as many participants as you want. However, to each participant you can only build one active link. You can change your link at any point in time. The participants to whom you have built an active link will be called your actively reached participants.

In addition to your active links, your so-called passive links are also relevant for you. A passive link for you is a link that another participant has built to you. That is, it is a participant's active link to you.

A participant cannot build links to himself. 


\begin{tabular}{ll}
\hline Type of link & Meaning \\
\hline 1. active & Your link to another participant. \\
& Graphical illustration: \\
\hline 2. passive & Other participant's link to you. \\
& Graphical illustration:
\end{tabular}

If you have an active and a passive link to a participant at the same time, it will be displayed as a double arrow:

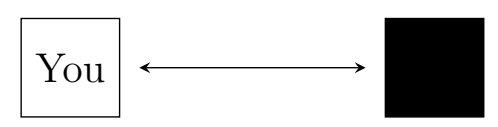

Building links: You see yourself as participant $T 1$ marked in red on the screen. You can build active links to other participants by writing the participant's number $(2,3,4$, 5 , or 6) into the upper line of your input mask labeled with "alternative or participant's number." Then press the enter key to switch to the lower line of the mask labeled with "X or Y 0 or $1 . "$

Enter "1" and confirm by pressing the enter key again. You can delete an existing link by writing the number of the respective participant on the upper line of the input mask and entering a "0" on the lower line.

As soon as you have built an active link, all participants in your group see this link on their screen. Likewise you can see all active links that currently exist in your group. When you are connected to another participant (actively or passively) this participant will be marked blue on your screen.

Choice of Alternative " $X$ " or Alternative " $Y$ ". Aside from choosing your links you also have to decide between the two alternatives "X" and "Y." The calculation of your payment depends on the alternative you and the participants to whom you are connected have chosen. It is not possible to choose between "X" and "Y" per link but you have to make a decision between the alternatives at every point of time, which then is valid for all links you have.

You choose your alternative "X" or "Y" similar to the choice of your active links by writing the character "a" for "choice of alternative" into the upper line of your input mask labeled with "alternative or participant's number." Then press the enter key to switch to the lower line of the mask labeled with "X or $\mathrm{Y} \quad 0$ or 1." Enter "X" or "Y" and confirm by pressing the enter key again.

At the beginning of the experiment you have to decide for each possible link (link to T2, T3, T4, T5, T6) whether you want to build it (enter "1") or not (enter "0"). Furthermore you have to enter one of the alternatives "X" or "Y." All inputs have to be confirmed by pressing the enter key. The clock is activated only after all participants 
made their decisions on their possible links and alternatives. When the time begins to run you will see your costs, earnings, and your accumulated profits. Please keep in mind that you may change your active links and your chosen alternative at any time during the whole period of 30 minutes.

Costs and Earnings. In this section you learn how your profit is determined.

You obtain a continuous profit stream, which is calculated in ExCU per minute. The size of your profit stream depends on your current active links and on the actions currently chosen by you and the participants to whom you are connected. It is calculated as follows.

Costs: Each active link that you have chosen at a point in time costs you 30 [50, 70] ExCU per minute. Thus, if you have a link for a whole minute you pay 30 [50, 70] ExCU. If you delete this link for example after 30 seconds you have to pay $15[25,35]$ ExCU. Your costs are subtracted continuously depending on the number of your current active links. You can see your costs per minute on the lower right corner of your screen. The costs are directly included in the payoff flow. Your passive links do not cause costs for you. That is, each link is paid by the player from whom the arrow points away. In the case of double $(\longleftrightarrow)$ arrows this means, that both concerned players have to pay.

Earnings: Your earnings are determined by the number of participants to whom you are connected, by the alternative you have chosen, and by the chosen alternatives of the participants to whom you are connected.

For each participant to whom you are actively or passively connected, you obtain an earnings flow (in ExCU per minute) that is determined according to the following table:

The other participant The other participant

\begin{tabular}{lcc} 
& chooses "X" & chooses "Y" \\
\hline You choose "X" & 20 & 80 \\
\hline You choose "Y" & 40 & 60 \\
\hline
\end{tabular}

The numbers listed in the table are given in ExCU per minute. Altogether, you obtain the sum of earnings flows from all participants to whom you are connected.

Suppose you have chosen "X" and you are connected to two participants. One of them has chosen "X" and the other has chosen "Y." Then you achieve an earnings flow of $20+80=100$ ExCU per minute.

Profit Flow: Your profit flow is the difference of your earnings flow and your cost flow from active links: Profit flow $=$ Earnings flow - Cost flow from active links

Accumulated profits: Your current accumulated profits are determined by continuously adding your profit flow. The accumulated profits are shown at the lower right corner of the screen. They are continuously updated according to your current earning and cost flows.

Example: Suppose you are confronted with an earnings flow of $80[100,120]$ ExCU per minute and a cost flow of $30[50,70]$ ExCU per minute. Then your accumulated 
profits grow in one minute by 50 ExCU. Your accumulated profits are continuously updated in this minute. If you or another participant of your group changes a link or the chosen alternative, for example, after 10 seconds, such that you now have a profit flow of 20 ExCU per minute then your accumulated profits will grow by 50 ExCU/60*10 $=8.33 \mathrm{ExCU}$ during the first 10 seconds and afterwards with a rate equal to $20 \mathrm{ExCU}$ per minute. Therefore, your accumulated profits would have grown in one minute by 8.33 $\mathrm{ExCU}+20 \mathrm{ExCU} / 60 * 50=8.33 \mathrm{ExCU}+16.67 \mathrm{ExCU}=25 \mathrm{ExCU}$.

Information. At any point in time the current values of the following variables will be displayed on the screen: Your current earnings flow (in ExCU per minute), your current cost flow (in ExCU per minute), your current profit flow (in ExCU per minute), your accumulated profits (in ExCU), your active links (as arrows in the graphical illustration on the screen and as "0" or "1" in a table), all active links in your group (as arrows in the graphical illustration), the participants to whom you are connected (actively or passively) are displayed as blue colored squares in the graphical illustration, the participants to whom you are not connected are displayed as gray colored squares in the graphical illustration, the chosen alternative of each participant (as characters " $\mathrm{X}$ " or "Y" beside the participants' squares in the graphical illustration), the amount of time the experiment is already running.

Payment. You obtain an initial endowment of 500 ExCU. The total amount of ExCU that is flowing to you during the 30 Minutes will be added to your initial endowment. It will be converted into Euro and will be paid out in cash immediately after the experiment is finished. The conversion rate is $400[300,170]$ ExCU to 1 Euro. The payment will be made individually and anonymous.

Before the experiment starts, you will be asked some questions about the rules of this game on your screen. If there is anything you do not understand, give us a sign. Your questions will be answered directly at your seat. When you have answered all questions the experiment starts.

Please look only at your own screen, do not talk to other participants, and do not leave the experimental program, which is running on your PC. 